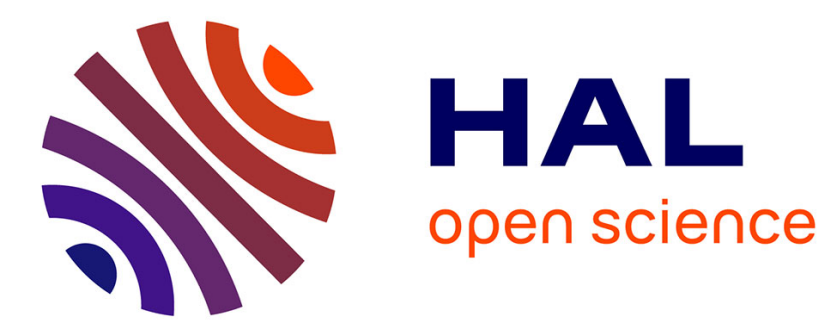

\title{
An optimisation approach for stability analysis and controller synthesis of linear hyperbolic systems
}

Pierre-Olivier Lamare, Antoine Girard, Christophe Prieur

\section{To cite this version:}

Pierre-Olivier Lamare, Antoine Girard, Christophe Prieur. An optimisation approach for stability analysis and controller synthesis of linear hyperbolic systems. ESAIM: Control, Optimisation and Calculus of Variations, 2016, 22 (4), pp.1236-1263. 10.1051/cocv/2016038 . hal-01331730

\author{
HAL Id: hal-01331730 \\ https://hal.science/hal-01331730
}

Submitted on 14 Jun 2016

HAL is a multi-disciplinary open access archive for the deposit and dissemination of scientific research documents, whether they are published or not. The documents may come from teaching and research institutions in France or abroad, or from public or private research centers.
L'archive ouverte pluridisciplinaire HAL, est destinée au dépôt et à la diffusion de documents scientifiques de niveau recherche, publiés ou non, émanant des établissements d'enseignement et de recherche français ou étrangers, des laboratoires publics ou privés. 


\title{
AN OPTIMISATION APPROACH FOR STABILITY ANALYSIS AND CONTROLLER SYNTHESIS OF LINEAR HYPERBOLIC SYSTEMS *
}

\author{
Pierre-Olivier Lamare ${ }^{1}$, Antoine Girard ${ }^{2}$ and Christophe Prieur ${ }^{3}$
}

\begin{abstract}
In this paper, we consider the problems of stability analysis and control synthesis for first-order hyperbolic linear Partial Differential Equations (PDEs) over a bounded interval with spatially varying coefficients. We propose Linear Matrix Inequalities (LMI) conditions for the stability and for the design of boundary and distributed control for the system. These conditions involve an infinite number of LMI to solve. Hence, we show how to overapproximate these constraints using polytopic embeddings to reduce the problem to a finite number of LMI. We show the effectiveness of the overapproximation with several examples and with the Saint-Venant equations with friction.
\end{abstract}

1991 Mathematics Subject Classification. 49J20,37N35,93B52.

\section{INTRODUCTION}

We consider the following general system

$$
\partial_{t} y(t, x)+\Lambda(x) \partial_{x} y(t, x)=F(x) y(t, x),
$$

where $t \in \mathbb{R}^{+}$is the time variable, $x \in[0,1]$ is the spatial variable, $y: \mathbb{R}^{+} \times[0,1] \rightarrow \mathbb{R}^{n}, F$ and $\Lambda$ are in $\mathrm{C}^{0}\left([0,1] ; \mathbb{R}^{n \times n}\right)$. The matrix $\Lambda(x)$ is diagonal and in addition

$$
\Lambda(x)=\left[\begin{array}{ccc}
\lambda_{1}(x) & & 0 \\
0 & \ddots & \\
& & \lambda_{n}(x)
\end{array}\right],
$$

with $\lambda_{k}(x)<0$ for $k \in\{1, \ldots, m\}$ and $\lambda_{k}(x)>0$ for $k \in\{m+1, \ldots, n\}$, for all $x \in[0,1]$. Let us introduce the following notation

$$
y=\left[y^{-}(t, x), y^{+}(t, x)\right]^{\top}
$$

Keywords and phrases: Hyperbolic PDE, Lyapunov method, LMI

* THIS WORK HAS BEEN PARTIALLY SUPPORTED BY THE LABEX PERSYVAL-LAB

ANR-11-LABX-0025.

1 BIOCORE project-team, Inria Sophia Antipolis - Méditerranée, 2004 route des Lucioles, BP 93, 06902 Sophia Antipolis Cedex, France; e-mail: pierre-olivier.lamare@inria.fr

2 Laboratoire des signaux et systèmes (L2S), CNRS, Centrale Supélec, Université Paris-Sud, Université Paris-Saclay, 3, rue Joliot-Curie, 91192 Gif-sur-Yvette, cedex, France; e-mail: antoine.girard@12s.centralesupelec.fr

${ }^{3}$ Department of Automatic Control, Gipsa-lab, 11 rue des Mathématiques, BP 46, 38402 Saint Martin d'Hères Cedex, France; e-mail: christophe.prieur@gipsa-lab.fr 
where $y^{-}: \mathbb{R}^{+} \times[0,1] \rightarrow \mathbb{R}^{m}$ and $y^{+}: \mathbb{R}^{+} \times[0,1] \rightarrow \mathbb{R}^{(n-m)}$. We consider the following boundary conditions

$$
\left[\begin{array}{l}
y^{-}(t, 1) \\
y^{+}(t, 0)
\end{array}\right]=G\left[\begin{array}{l}
y^{-}(t, 0) \\
y^{+}(t, 1)
\end{array}\right], \quad t \in \mathbb{R}^{+}
$$

where $G$ is a matrix in $\mathbb{R}^{n \times n}$. The initial condition is

$$
y(0, x)=y^{0}(x), \quad x \in(0,1),
$$

where $y^{0} \in \mathrm{L}^{2}\left((0,1) ; \mathbb{R}^{n}\right)$. It can be shown that the following result holds, see [10] and the references therein.

Proposition 1.1. There exists a unique (weak) solution $y \in \mathrm{C}^{0}\left(\mathbb{R}^{+} ; \mathrm{L}^{2}\left((0,1) ; \mathbb{R}^{n}\right)\right)$ to the Cauchy problem (1)-(3).

Let us define the concept of Global Exponential Stability (GES) for system (1)-(3).

Definition 1.2. The system (1)-(3) is said to be Globally Exponentially Stable if there exist $\nu>0$ and $C>0$ such that, for every initial condition $y^{0} \in \mathrm{L}^{2}\left((0,1) ; \mathbb{R}^{n}\right)$, the solution to the Cauchy problem (1)-(3) satisfies

$$
|y(t, \cdot)|_{\mathrm{L}^{2}\left((0,1) ; \mathbb{R}^{n}\right)} \leq C e^{-\nu t}\left|y^{0}\right|_{\mathrm{L}^{2}\left((0,1) ; \mathbb{R}^{n}\right)}, \forall t \in \mathbb{R}^{+} .
$$

While for finite dimensional and time-delay systems a large number of numerical techniques for stability analysis exists, for PDEs these tools are mostly lacking. In this paper, we propose some techniques to verify numerically the existence of quadratic Lyapunov functions for first-order hyperbolic PDEs over a bounded interval with spatially varying coefficients. Besides this analysis aspect, we propose some techniques for the synthesis of boundary and distributed controls.

The Lyapunov analysis has been a common and powerful approach to analyse and control systems in finite dimension for several decades. Among the large literature on stability analysis and stabilisation of finite dimensional system let us mention [3] covering a large number of results on the resolution of Linear Matrix Inequalities (LMI) coming from the Lyapunov analysis. In [14], a numerical toolbox to solve various control problems, in particular those written in terms of LMI, is presented. It allows in particular an optimisation of any given convex cost (such as the trace of the Lyapunov matrix). Thus, it is particularly attractive to express problem under LMI form to take advantages of the existing toolboxes.

The use of Lyapunov function is now appearing for hyperbolic systems. Lyapunov converse results have been stated recently in [12]. More specifically, a special attention has been made on quadratic Lyapunov functions. Indeed, this class of functions allows to express conditions for stability as Matrix Inequalities (MI). We can cite also [5], [10], [15], and [17] for the linear case. LMI conditions derived by an operator approach is used in [11] for the $H^{\infty}$ boundary control of parabolic and hyperbolic systems. Quadratic Lyapunov function has also been used for $2 \times 2$ quasilinear systems [7] and $n \times n$ quasilinear systems [6]. MI-based conditions derived from a quadratic Lyapunov function were stated in [4] for the construction of boundary observers for linear as well as for quasilinear hyperbolic systems. However, the approach by a quadratic Lyapunov function is not always effective to prove stability for hyperbolic systems. A result from [1] gives a necessary and sufficient condition for the existence of a quadratic Lyapunov function. In [9], an example with a stabilising static output feedback has been designed such that the previous condition is violated and thus that there does not exist a quadratic Lyapunov function for this system.

The results in this paper are related to the resolution of the LMIs proposed in [15] and to the control synthesis. We also propose a Lyapunov function with an "affine" kernel similar to the one used in [8]. The conditions involve the spatial variable, hence the number of constraints is infinite. These conditions are analogous to stability conditions for finite-dimensional Linear Parameter Varying (LPV) systems. Hence, an approach inspired by this framework is applied to find a candidate Lyapunov function. More precisely, to reduce the numerical complexity, different approximations based on properties of the exponential and affine functions are considered in this paper. The control synthesis relies on a combination of classical techniques coming from the stabilisation 
for discrete and continuous-time finite dimensional systems. Then, the latter overapproximation techniques are used to get a finite number of conditions.

The paper is organized as follows. In Section 2, we propose new LMI conditions for the stability of systems described by (1)-(3). In Section 3, we address the problem of the design of static boundary and distributed controllers. In Section 4, overapproximation techniques are presented to reduce the complexity of the LMI conditions stated so far. Finally, in Section 5, methods are tested on academic examples and with the SaintVenant equations with friction.

Notation. The set of complex numbers is denoted by $\mathbb{C}$. The set of square real matrices of dimension $n$ is denoted by $\mathbb{R}^{n \times n}$. Given a matrix $A$, the transpose of the matrix $A$ is denoted by $A^{\top}$. Given $N$ square matrices $A_{1}, \ldots, A_{N}$, of respective dimension $k_{1}, \ldots, k_{N}$, the block diagonal matrix $A \in \mathbb{R}^{\left(k_{1}+\cdots+k_{N}\right) \times\left(k_{1}+\cdots+k_{N}\right)}$ whose block diagonal matrices are $A_{1}, \ldots, A_{N}$, is denoted by $\operatorname{diag}\left[A_{1}, \ldots, A_{N}\right]$. The identity matrix of dimension $n$ is denoted by $I_{n}$. For a symmetric matrix $A \in \mathbb{R}^{n \times n}, A$ being positive definite is denoted by $A>0$, while $A$ being positive semi-definite is denoted by $A \geq 0$. The smallest eigenvalue of a matrix $A \in \mathbb{R}^{n \times n}$ is denoted by $\lambda_{\min }(A)$ while its largest eigenvalue is denoted by $\lambda_{\max }(A)$. The derivative of a matrix $A(x)$ with respect to variable $x$ is denoted by $A^{\prime}(x)$.

\section{Conditions for Stability}

In this section, we propose a Lyapunov function, and derive some conditions for the solution to system (1)-(3) to satisfy (4).

Let us introduce the following notation

$$
\Lambda(x)=\operatorname{diag}\left[\Lambda^{-}(x), \Lambda^{+}(x)\right],
$$

where $\Lambda^{-}(x)=\operatorname{diag}\left[\lambda_{1}(x), \ldots, \lambda_{m}(x)\right]$ and $\Lambda^{+}(x)=\operatorname{diag}\left[\lambda_{m+1}(x), \ldots, \lambda_{n}(x)\right]$. Let us denote $|\Lambda(x)|$ the matrix whose elements are the absolute value of the elements of the matrix $\Lambda(x)$, that is

$$
|\Lambda(x)|=\operatorname{diag}\left[-\Lambda^{-}(x), \Lambda^{+}(x)\right],
$$

and let us denote by $\tilde{I}_{n, m}$ the matrix

$$
\tilde{I}_{n, m}=\left[\begin{array}{cc}
-I_{m} & 0_{m, n-m} \\
0_{n-m, m} & I_{n-m}
\end{array}\right]
$$

For a matrix $A$ in $\mathbb{R}^{n \times n}$, we decompose it in four block matrices $A_{--}$in $\mathbb{R}^{m \times m}, A_{-+}$in $\mathbb{R}^{m \times(n-m)}, A_{+-}$in $\mathbb{R}^{(n-m) \times m}$, and $A_{++}$in $\mathbb{R}^{(n-m) \times(n-m)}$ such that $A=\left[\begin{array}{ll}A_{--} A_{-+} \\ A_{+-} & A_{++}\end{array}\right]$.

\section{Exponential Kernel.}

In [15], sufficient conditions have been given for the stability of (1)-(3) with $\Lambda(x)$ and $F(x)$ constant. We consider a slightly different Lyapunov function

$$
V(y)=\int_{0}^{1} y^{\top}(x)|\Lambda(x)|^{-1} \mathcal{Q}(x) y(x) d x
$$

where

$$
\mathcal{Q}(x)=\operatorname{diag}\left[e^{2 \mu x} Q^{-}, e^{-2 \mu x} Q^{+}\right],
$$

with $Q^{-}$in $\mathbb{R}^{m \times m}, Q^{+}$in $\mathbb{R}^{(n-m) \times(n-m)}$ are two symmetric positive definite matrices and $\mu$ a real coefficient. 
Proposition 2.1. If there exist $\nu>0, \mu$ in $\mathbb{R}$, and symmetric positive definite matrices $Q^{-}$in $\mathbb{R}^{m \times m}$ and $Q^{+}$ in $\mathbb{R}^{(n-m) \times(n-m)}$ such that for $\mathcal{Q}(x)$ given by (8) the following conditions hold, for all $x \in[0,1]$,

$$
\begin{aligned}
& \mathcal{Q}(x) \Lambda(x)=\Lambda(x) \mathcal{Q}(x), \\
& -2 \mu \mathcal{Q}(x)+F^{\top}(x)|\Lambda(x)|^{-1} \mathcal{Q}(x)+\mathcal{Q}(x)|\Lambda(x)|^{-1} F(x) \leq-2 \nu|\Lambda(x)|^{-1} \mathcal{Q}(x),
\end{aligned}
$$

together with

$$
\left[\begin{array}{cc}
I_{m} & 0_{m, n-m} \\
G_{+-} & G_{++}
\end{array}\right]^{\top} \tilde{I}_{n, m} \mathcal{Q}(0)\left[\begin{array}{cc}
I_{m} & 0_{m, n-m} \\
G_{+-} & G_{++}
\end{array}\right] \leq\left[\begin{array}{cc}
G_{--} & G_{-+} \\
0_{n-m, m} & I_{n-m}
\end{array}\right]^{\top} \tilde{I}_{n, m} \mathcal{Q}(1)\left[\begin{array}{cc}
G_{--} & G_{-+} \\
0_{n-m, m} & I_{n-m}
\end{array}\right],
$$

then the linear hyperbolic system (1)-(3) is GES.

Proof. For the stability analysis we consider the candidate Lyapunov function $(7)$, where $\mathcal{Q}(x)$ is given by $(8)$. Let us introduce the constants

$$
\begin{aligned}
& \underline{\lambda}=\min _{x \in[0,1]} \lambda_{\min }\left(|\Lambda(x)|^{-1} \mathcal{Q}(x)\right), \\
& \bar{\lambda}=\max _{x \in[0,1]} \lambda_{\max }\left(|\Lambda(x)|^{-1} \mathcal{Q}(x)\right) .
\end{aligned}
$$

The matrix $|\Lambda(x)|^{-1} \mathcal{Q}(x)$ being symmetric positive definite (due to condition (9) and since $\mathcal{Q}(x)$ and $|\Lambda(x)|^{-1}$ are symmetric positive definite), one can conclude that $\bar{\lambda}, \underline{\lambda}>0$ and for all $y \in \mathrm{L}^{2}\left((0,1) ; \mathbb{R}^{n}\right)$,

$$
\underline{\lambda}|y|_{\mathrm{L}^{2}\left((0,1) ; \mathbb{R}^{n}\right)}^{2} \leq V(y) \leq \bar{\lambda}|y|_{\mathrm{L}^{2}\left((0,1) ; \mathbb{R}^{n}\right)}^{2} .
$$

Let us compute the time-derivative of the candidate Lyapunov function (7) along the solutions to system (1), (2). Using the commutativity condition (9), we have

$$
\begin{aligned}
\dot{V} & =2 \int_{0}^{1} y_{t}^{\top}(t, x)|\Lambda(x)|^{-1} \mathcal{Q}(x) y(t, x) d x \\
& =-2 \int_{0}^{1} y^{\top}(t, x) \tilde{I}_{n, m} \mathcal{Q}(x) y_{x}(t, x) d x+2 \int_{0}^{1} y^{\top}(t, x) \mathcal{Q}(x)|\Lambda(x)|^{-1} F(x) y(t, x) d x .
\end{aligned}
$$

Noting that $-2 y^{\top} \tilde{I}_{n, m} \mathcal{Q} y_{x}=-\left(y^{\top} \tilde{I}_{n, m} \mathcal{Q} y\right)_{x}+y^{\top} \tilde{I}_{n, m} \mathcal{Q}^{\prime} y$ and $\tilde{I}_{n, m} \mathcal{Q}^{\prime}=-2 \mu \mathcal{Q}$, one has

$$
\begin{aligned}
\dot{V}= & -\left[y^{\top}(t, x) \tilde{I}_{n, m} \mathcal{Q}(x) y(t, x)\right]_{0}^{1}-2 \mu \int_{0}^{1} y^{\top}(t, x) \mathcal{Q}(x) y(t, x) d x \\
& +2 \int_{0}^{1} y^{\top}(t, x) \mathcal{Q}(x)|\Lambda(x)|^{-1} F(x) y(t, x) d x \\
= & {\left[\begin{array}{cc}
y^{-}(t, 0) \\
y^{+}(t, 1)
\end{array}\right]^{\top}\left[\begin{array}{cc}
I_{m} & 0_{m, n-m} \\
G_{+-} & G_{++}
\end{array}\right]^{\top} \tilde{I}_{n, m} \mathcal{Q}(0)\left[\begin{array}{cc}
I_{m} & 0_{m, n-m} \\
G_{+-} & G_{++}
\end{array}\right] } \\
& \left.-\left[\begin{array}{cc}
G_{--} & G_{-+} \\
0_{n-m, m} & I_{n-m}
\end{array}\right]^{\top} \tilde{I}_{n, m} \mathcal{Q}(1)\left[\begin{array}{cc}
G_{--} & G_{-+} \\
0_{n-m, m} & I_{n-m}
\end{array}\right]\right]\left[\begin{array}{c}
y^{-}(t, 0) \\
y^{+}(t, 1)
\end{array}\right] \\
& +2 \int_{0}^{1} y^{\top}(t, x) \mathcal{Q}(x)|\Lambda(x)|^{-1} F(x) y(t, x) d x-2 \mu \int_{0}^{1} y^{\top}(t, x) \mathcal{Q}(x) y(t, x) d x .
\end{aligned}
$$

Then, (10) and (11) imply that $\dot{V} \leq-2 \nu V$. Hence, for all $t \in \mathbb{R}^{+}$, one has $V(y(t, \cdot)) \leq e^{-2 \nu t} V\left(y^{0}\right)$. Combining this relation with (14), the proof is complete. 
Remark 2.2. In the proof of Proposition 2.1, for the computation of the time derivative of $V$, we have proceeded as if the solutions were in $\mathrm{C}^{1}$. Nonetheless, the calculus remains valid with $\mathrm{L}^{2}$-solutions. It is due to the density of $\mathrm{C}^{1}$-solutions in the set of $\mathrm{L}^{2}$-solutions, see [2, Page 67, Section 2.1] for a developed explanation.

The relation between the previous stability conditions and LPV systems is done in the following proposition.

Proposition 2.3. Let $\mu$ in $\mathbb{R}$. Conditions (10) and (11) are satisfied if and only if the continuous time LPV system

$$
\dot{p}(t)=\operatorname{diag}\left[e^{2 \mu x} I_{m}, e^{-2 \mu x} I_{n-m}\right]\left(|\Lambda(x)|^{-1} F(x)-\mu I_{n}\right) p(t), \quad x \in[0,1],
$$

and the discrete time system

$$
h(t+1)=\operatorname{diag}\left[I_{m}, e^{-\mu} I_{n-m}\right] e^{\mu} G \operatorname{diag}\left[I_{m}, e^{\mu} I_{n-m}\right] h(t)
$$

share a common block diagonal Lyapunov matrix diag $\left[Q^{-}, Q^{+}\right]$, where $Q^{-}$and $Q^{+}$are symmetric matrices in $\mathbb{R}^{m \times m}$ and $\mathbb{R}^{(n-m) \times(n-m)}$ respectively.

Proof. Condition (10) describes a condition for the stability of the continuous time LPV system (15) via the existence of the Lyapunov matrix diag $\left[Q^{-}, Q^{+}\right]$.

Condition (11) may be developed as

$$
P=\left[\begin{array}{ll}
P_{--} & P_{-+} \\
P_{+-} & P_{++}
\end{array}\right] \leq 0,
$$

with

$$
\begin{aligned}
& P_{--}=e^{2 \mu} G_{--}^{\top} Q^{-} G_{--}+G_{+-}^{\top} Q^{+} G_{+-}-Q^{-}, \\
& P_{-+}=e^{2 \mu} G_{--}^{\top} Q^{-} G_{-+}+G_{+-}^{\top} Q^{+} G_{++}, \\
& P_{+-}=P_{-+}^{\top}, \\
& P_{++}=e^{2 \mu} G_{-+}^{\top} Q^{-} G_{-+}+G_{++}^{\top} Q^{+} G_{++}-e^{-2 \mu} Q^{+} .
\end{aligned}
$$

The matrix $P$ in (17) may be rewritten as

$$
P=\left(e^{\mu} G\right)^{\top} \operatorname{diag}\left[Q^{-}, e^{-2 \mu} Q^{+}\right] e^{\mu} G-\operatorname{diag}\left[Q^{-}, e^{-2 \mu} Q^{+}\right]
$$

Thus, with (18), inequality (17) leads to establish

$$
\begin{aligned}
P \leq 0 \Leftrightarrow & e^{\mu} G^{\top} \operatorname{diag}\left[I_{m}, e^{-\mu} I_{n-m}\right] \operatorname{diag}\left[Q^{-}, Q^{+}\right] \operatorname{diag}\left[I_{m}, e^{-\mu} I_{n-m}\right] e^{\mu} G \\
& \leq \operatorname{diag}\left[I_{m}, e^{-\mu} I_{n-m}\right] \operatorname{diag}\left[Q^{-}, Q^{+}\right] \operatorname{diag}\left[I_{m}, e^{-\mu} I_{n-m}\right] \\
\Leftrightarrow & \operatorname{diag}\left[I_{m}, e^{\mu} I_{n-m}\right]\left(e^{\mu} G\right)^{\top} \operatorname{diag}\left[I_{m}, e^{-\mu} I_{n-m}\right] \operatorname{diag}\left[Q^{-}, Q^{+}\right] \\
& \times \operatorname{diag}\left[I_{m}, e^{-\mu} I_{n-m}\right] e^{\mu} G \operatorname{diag}\left[I_{m}, e^{\mu} I_{n-m}\right] \leq \operatorname{diag}\left[Q^{-}, Q^{+}\right] .
\end{aligned}
$$

Hence, the discrete-time system (16) shares a common block diagonal Lyapunov matrix diag $\left[Q^{-}, Q^{+}\right]$with the continuous-time LPV system (15). It concludes the proof of Proposition 2.3.

Remark 2.4. A consequence of Proposition 2.3 is a trade-off in the choice of $\mu$ between the satisfaction of (10) and (11). Positive or negative values of $\mu$ allow to consider (15) more stable or unstable respectively, but have the reverse effect on (16). 


\section{Affine Kernel.}

Other sufficient conditions for the global exponential stability of system (1)-(3) are obtained when considering a different kernel $\mathcal{Q}(x)$ in (7). The next proposition is built using

$$
\mathcal{Q}(x)=\operatorname{diag}\left[(1+\mu x) Q^{-},(1-\mu x) Q^{+}\right],
$$

in (7).

Proposition 2.5. If there exist $\nu>0, \mu \in(-1,1)$, and symmetric positive definite matrices $Q^{-}$in $\mathbb{R}^{m \times m}$ and $Q^{+}$in $\mathbb{R}^{(n-m) \times(n-m)}$ such that for $\mathcal{Q}(x)$ given by (19) the following conditions hold, for all $x \in[0,1]$,

$$
\begin{aligned}
& \mathcal{Q}(x) \Lambda(x)=\Lambda(x) \mathcal{Q}(x), \\
& -\mu \mathcal{Q}(0)+F^{\top}(x)|\Lambda(x)|^{-1} \mathcal{Q}(x)+\mathcal{Q}(x)|\Lambda(x)|^{-1} F(x) \leq-2 \nu|\Lambda(x)|^{-1} \mathcal{Q}(x),
\end{aligned}
$$

together with

$$
\left[\begin{array}{cc}
I_{m} & 0_{m, n-m} \\
G_{+-} & G_{++}
\end{array}\right]^{\top}\left[\begin{array}{cc}
-Q^{-} & 0_{m, n-m} \\
0_{n-m, m} & Q^{+}
\end{array}\right]\left[\begin{array}{cc}
I_{m} & 0_{m, n-m} \\
G_{+-} & G_{++}
\end{array}\right] \leq\left[\begin{array}{cc}
G_{--} & G_{-+} \\
0_{n-m, m} & I_{n-m}
\end{array}\right]^{\top}\left[\begin{array}{cc}
-(1+\mu) Q^{-} & 0 \\
0 & (1-\mu) Q^{+}
\end{array}\right]\left[\begin{array}{cc}
G_{--} & G_{-+} \\
0_{n-m, m} & I_{n-m}
\end{array}\right],
$$

then the linear hyperbolic system (1)-(3) is GES.

Proof. As mentioned earlier, we consider the candidate Lyapunov function (7) where $\mathcal{Q}(x)$ is given by (19). We used the definitions of $\underline{\lambda}$ and $\bar{\lambda}$ given in (12) and (13) in the context of $\mathcal{Q}(x)$ given by (19). The matrix $|\Lambda(x)|^{-1} \mathcal{Q}(x)$ is positive definite (due to condition (20)), one can conclude that $\bar{\lambda}, \underline{\lambda}>0$. Therefore, for all $y \in \mathrm{L}^{2}\left((0,1) ; \mathbb{R}^{n}\right)$,

$$
\underline{\lambda}|y|_{\mathrm{L}^{2}\left((0,1) ; \mathbb{R}^{n}\right)}^{2} \leq V(y) \leq \bar{\lambda}|y|_{\mathrm{L}^{2}\left((0,1) ; \mathbb{R}^{n}\right)}^{2} .
$$

Let us compute the time-derivative of the candidate Lyapunov function along the solutions to system (1), (2). Using the commutativity condition (20), we have

$$
\begin{aligned}
& \dot{V}=2 \int_{0}^{1} y_{t}^{\top}(t, x)|\Lambda(x)|^{-1} \mathcal{Q}(x) y(t, x) d x \\
& =-2 \int_{0}^{1} y_{x}^{\top}(t, x)\left[\begin{array}{cc}
-I_{m} & 0_{m, n-m} \\
0_{n-m, m} & I_{n-m}
\end{array}\right] \mathcal{Q}(x) y(t, x) d x+2 \int_{0}^{1} y^{\top}(t, x) \mathcal{Q}(x)|\Lambda(x)|^{-1} F(x) y(t, x) d x \\
& =-2 \int_{0}^{1} y_{x}^{\top}(t, x) \mathcal{P}(x) y(t, x) d x+2 \int_{0}^{1} y^{\top}(t, x) \mathcal{Q}(x)|\Lambda(x)|^{-1} F(x) y(t, x) d x,
\end{aligned}
$$


where $\mathcal{P}(x)=\left[\begin{array}{cc}-(1+\mu x) Q^{-} & 0_{m, n-m} \\ 0_{m, n-m} & (1-\mu x) Q^{+}\end{array}\right]$. Noting that $-2 y_{x}^{\top} \mathcal{P} y=-\left(y^{\top} \mathcal{P} y\right)_{x}+y^{\top} \mathcal{P}^{\prime} y$ we get

$$
\begin{aligned}
\dot{V}= & -\left[y^{\top}(t, x) \mathcal{P}(x) y(t, x)\right]_{0}^{1}-\mu \int_{0}^{1} y^{\top}(t, x) \mathcal{Q}(0) y(t, x) d x \\
& +2 \int_{0}^{1} y^{\top}(t, x) \mathcal{Q}(x)|\Lambda(x)|^{-1} F(x) y(t, x) d x \\
= & {\left[\begin{array}{ll}
y^{-}(t, 0) \\
y^{+}(t, 1)
\end{array}\right]^{\top}\left[\left[\begin{array}{cc}
I_{m} & 0 \\
G_{+-} & G_{++}
\end{array}\right]^{\top} \mathcal{P}(0)\left[\begin{array}{cc}
I_{m} & 0_{m, n-m} \\
G_{+-} & G_{++}
\end{array}\right]\right.} \\
& \left.-\left[\begin{array}{cc}
G_{--} & G_{-+} \\
0_{n-m, m} & I_{n-m}
\end{array}\right]^{\top} \mathcal{P}(1)\left[\begin{array}{cc}
G_{--} & G_{-+} \\
0_{n-m, m} & I_{n-m}
\end{array}\right]\right]\left[\begin{array}{c}
y^{-}(t, 0) \\
y^{+}(t, 1)
\end{array}\right] \\
& -\mu \int_{0}^{1} y^{\top}(t, x) \mathcal{Q}(0) y(t, x) d x+2 \int_{0}^{1} y^{\top}(t, x) \mathcal{Q}(x)|\Lambda(x)|^{-1} F(x) y(t, x) d x
\end{aligned}
$$

Then, (21) and (22) imply that $\dot{V} \leq-2 \nu V$. Hence, for all $t \in \mathbb{R}^{+}$, one has $V(y(t, \cdot)) \leq e^{-2 \nu t} V\left(y^{0}\right)$. Combining this relation with (23) the proof is complete.

\section{Remark 2.6. The condition}

$$
\mathcal{Q}(x) \Lambda(x)=\Lambda(x) \mathcal{Q}(x)
$$

holds as soon as $Q^{-}$and $Q^{+}$are diagonal (as in [10]) but this is not necessary. It becomes necessary when the eigenvalues of $\Lambda(x)$ are of multiplicity 1. It may happen that the matrices cannot be chosen diagonal even in the constant case. For instance, let us consider the following matrices in $\mathbb{R}^{2 \times 2}$

$$
\begin{aligned}
\Lambda & =I_{2}, \\
F & =\left[\begin{array}{cc}
-10 & -9 \\
10 & 6
\end{array}\right], \\
G & =\frac{1}{2} I_{2} .
\end{aligned}
$$

It may be proved that there do not exist a real $\mu$ and a diagonal matrix $Q$ in $\mathbb{R}^{2 \times 2}$ such that (10) and (11) hold (see Appendix A). However, there exist a real $\mu$ and a non-diagonal matrix $Q$ such that it satisfies (9), (10), and (11) as, for instance

$$
\begin{aligned}
\mu & =0.2, \\
Q & =\left[\begin{array}{ll}
3.3860 & 2.2424 \\
2.2424 & 3.1074
\end{array}\right] .
\end{aligned}
$$

\section{Controller Synthesis}

\subsection{Boundary Control Synthesis}

We consider next the problem of boundary control synthesis, when boundary condition (2) is given by

$$
G=T+L K_{B},
$$

where matrices $T$ in $\mathbb{R}^{n \times n}, L$ in $\mathbb{R}^{n \times q}(n>q)$ are given and the matrix $K_{B}$ in $\mathbb{R}^{q \times n}$ has to be designed such that system (1)-(3) with the boundary conditions (25) is GES. We propose results based on the use of the Lyapunov function (7) in the context of the exponential kernel (8) only. The case of the affine kernel is considered in Appendix B. 
Theorem 3.1. If there exist $\nu>0, \mu$ in $\mathbb{R}$, a matrix $U$ in $\mathbb{R}^{q \times n}$, and symmetric positive definite matrices $S^{-}$ in $\mathbb{R}^{m \times m}, S^{+}$in $\mathbb{R}^{(n-m) \times(n-m)}$ such that, with $S(x)=\operatorname{diag}\left[e^{-2 \mu x} S^{-}, e^{2 \mu x} S^{+}\right]$, the following conditions hold, for all $x \in[0,1]$,

$$
\begin{aligned}
& S(x) \Lambda(x)=\Lambda(x) S(x), \\
& -2 \mu S(x)+S(x) F^{\top}(x)|\Lambda(x)|^{-1}+|\Lambda(x)|^{-1} F(x) S(x) \leq-2 \nu S(x)|\Lambda(x)|^{-1}, \\
& {\left[\begin{array}{cc}
\operatorname{diag}\left[S^{-}, e^{-2 \mu} S^{+}\right] & (T S(0)+L U)^{\top} \\
T S(0)+L U & \operatorname{diag}\left[e^{-2 \mu} S^{-}, S^{+}\right]
\end{array}\right] \geq 0,}
\end{aligned}
$$

then the boundary control given by (25) with

$$
K_{B}=U S^{-1}(0)
$$

is such that system (1)-(3) is GES and a Lyapunov function of the form (7) exists with $\mathcal{Q}(x)=S^{-1}(x)$.

Proof. Replacing $U$ by $K_{B} S(0)$ and applying the Schur complement formula (see, for instance, [3, Pages 7-8]) in (28) one gets

$$
\operatorname{diag}\left[S^{-}, e^{-2 \mu} S^{+}\right]-S(0)\left(T+L K_{B}\right)^{\top} \operatorname{diag}\left[e^{-2 \mu} S^{-}, S^{+}\right]^{-1}\left(T+L K_{B}\right) S(0) \geq 0
$$

Reassembling the term in one matrix and multiplying from the left and right by $S^{-1}(0)$ we get a matrix

$$
M=\left[\begin{array}{ll}
M_{--} & M_{-+} \\
M_{+-} & M_{++}
\end{array}\right] \geq 0,
$$

with

$$
\begin{aligned}
M_{--}= & \left(S^{-}\right)^{-1}-e^{2 \mu}\left(T+L K_{B}\right)_{--}^{\top}\left(S^{-}\right)^{-1}\left(T+L K_{B}\right)_{--}-\left(T+L K_{B}\right)_{+-}^{\top}\left(S^{+}\right)^{-1}\left(T+L K_{B}\right)_{+-}, \\
M_{-+}= & -e^{2 \mu}\left(T+L K_{B}\right)_{--}^{\top}\left(S^{-}\right)^{-1}\left(T+L K_{B}\right)_{-+}-\left(T+L K_{B}\right)_{+-}^{\top}\left(S^{+}\right)^{-1}\left(T+L K_{B}\right)_{++}, \\
M_{+-}= & M_{-+}^{\top}, \\
M_{++}= & e^{-2 \mu}\left(S^{+}\right)^{-1}-e^{2 \mu}\left(T+L K_{B}\right)_{-+}^{\top}\left(S^{-}\right)^{-1}\left(T+L K_{B}\right)_{-+} \\
& -\left(T+L K_{B}\right)_{++}^{\top}\left(S^{+}\right)^{-1}\left(T+L K_{B}\right)_{++} .
\end{aligned}
$$

Letting $Q^{-}=\left(S^{-}\right)^{-1}, Q^{+}=\left(S^{+}\right)^{-1}$ we get

- condition (9) by multiplying left and right $(26)$ by $\mathcal{Q}(x)$,

- condition (10) by multiplying left and right $(27)$ by $\mathcal{Q}(x)$,

- condition (11) from the matrix $M$ in (31). Indeed, $M$ is equal to the matrix $-P$ in (17) in the proof of Proposition 2.3, this latter is equivalent to the inequality (11).

It concludes the proof of Theorem 3.1.

\subsection{Distributed Control Synthesis} form

We consider the case of a distributed control that is that the matrix in the right-hand side of (1) is of the

$$
F(x)=H(x)+B(x) K_{D}(x), \quad x \in[0,1],
$$

where matrices $H(x)$ in $\mathbb{R}^{n \times n}, B(x)$ in $\mathbb{R}^{n \times p}(n>p)$ are given, and matrix $K_{D}(x)$ in $\mathbb{R}^{p \times n}$ has to be designed such that system (1)-(3) is GES with the distributed control (32). In the following, we assume that $K_{D}(x)$ is 
given by

$$
K_{D}(x)=\sum_{i=1}^{\ell} \alpha_{i}(x) K_{i},
$$

where $\alpha_{i}, K_{i} i=1, \ldots, \ell$, are, respectively, some continuous real functions and matrices in $\mathbb{R}^{p \times n}$.

Remark 3.2. Examples of suitable functions $\alpha_{i}$, in (33), are the Bézier functions basis and spline basis functions of degree 1 .

Remark 3.3. Though most of the physical devices are controlled from the boundaries (see, for instance, [2] or [5]), there exist physical applications embedding such distributed controller (32). For instance, the evolution of a population of bacteria or animals in cultivation conditions may be described by a system of the form (1). As a matter of example, in [13], a hyperbolic PDE model of chemostat is considered. In such situation $y(t, x)$ represents the number of individuals of age $x$ at time $t$, the function $F$ is a mortality function or a sampling. Thus, the control may be to synthesise this mortality rate or this sampling, for instance to insure some criteria on the cultivation process. In the latter reference, it should be noted that the control quantity is a dilution rate and is time dependent contrary to $(32)$.

Henceforth, for a given $\mu$ in $\mathbb{R}$, let us denote by $I_{n, m}^{e}(x)$ the matrix

$$
I_{n, m}^{e}(x)=\operatorname{diag}\left[e^{2 \mu x} I_{m}, e^{-2 \mu x} I_{n-m}\right]
$$

Theorem 3.4. Let an integer $\ell>0$ be given. If there exist $\nu>0, \mu$ in $\mathbb{R}$, matrices $U_{i}$ in $\mathbb{R}^{p \times n}$, $i=1, \ldots, \ell$, and symmetric positive definite matrices $S^{-}$in $\mathbb{R}^{m \times m}, S^{+}$in $\mathbb{R}^{(n-m) \times(n-m)}$ such that, with $S(x)=\operatorname{diag}\left[e^{-2 \mu x} S^{-}, e^{2 \mu x} S^{+}\right]$, the following conditions hold, for all $x \in[0,1]$,

$$
\begin{aligned}
& S(x) \Lambda(x)=\Lambda(x) S(x), \\
& \left(|\Lambda(x)|^{-1} H(x)-\mu I_{n}\right) S(x)+S(x)\left(|\Lambda(x)|^{-1} H(x)-\mu I_{n}\right)^{\top} \\
& +\sum_{i=1}^{\ell} \alpha_{i}(x)\left(I_{n, m}^{e}(x)\right)^{-1} U_{i}^{\top} B^{\top}(x)|\Lambda(x)|^{-1}+\sum_{i=1}^{\ell} \alpha_{i}(x)|\Lambda(x)|^{-1} B(x) U_{i}\left(I_{n, m}^{e}(x)\right)^{-1} \\
& \leq-2 \nu S(x)|\Lambda(x)|^{-1}, \\
& {\left[\begin{array}{c}
\operatorname{diag}\left[S^{-}, e^{-2 \mu} S^{+}\right] \quad(G S(0))^{\top} \\
G S(0)
\end{array}\right] \geq 0,}
\end{aligned}
$$

with $I_{n, m}^{e}(x)$ given in (34), then the distributed control given by (32) and (33) with

$$
K_{i}=U_{i} S^{-1}(0), \quad i=1, \ldots, \ell
$$

is such that system (1)-(3) is GES and a Lyapunov function of the form (7) exists with $\mathcal{Q}(x)=S^{-1}(x)$.

Proof. We know that system (1)-(3) is exponentially stable if conditions of Proposition 2.1 hold. To apply this result let us check (9), (10), and (11) successively. Using the Schur complement formula with (37), letting $Q^{-}=\left(S^{-}\right)^{-1}$ and $Q^{+}=\left(S^{+}\right)^{-1}$ as in the proof of Theorem 3.1, conditions $(9)$ and (11) are satisfied. We can rewrite $(10)$ as

$$
\left(|\Lambda(x)|^{-1} F(x)-\mu I_{n}\right)^{\top} \mathcal{Q}(x)+\mathcal{Q}(x)\left(|\Lambda(x)|^{-1} F(x)-\mu I_{n}\right) \leq-2 \nu|\Lambda(x)|^{-1} \mathcal{Q}(x)
$$


We use the expression of $F$ given by (32), (33) and get

$$
\begin{aligned}
& \left(|\Lambda(x)|^{-1}\left(H(x)+B(x) \sum_{i=1}^{\ell} \alpha_{i}(x) K_{i}\right)-\mu I_{n}\right)^{\top} \mathcal{Q}(x) \\
& +\mathcal{Q}(x)\left(|\Lambda(x)|^{-1}\left(H(x)+B(x) \sum_{i=1}^{\ell} \alpha_{i}(x) K_{i}\right)-\mu I_{n}\right) \leq-2 \nu|\Lambda(x)|^{-1} \mathcal{Q}(x) .
\end{aligned}
$$

This last inequality is not convex in $K_{i}$ and $\mathcal{Q}(x)$. To overcome this issue we multiply (39) at the left and right by $S(x)$, we get

$$
\begin{aligned}
& \left(|\Lambda(x)|^{-1} H(x)-\mu I_{n}\right) S(x)+S(x)\left(|\Lambda(x)|^{-1} H(x)-\mu I_{n}\right)^{\top} \\
& +\sum_{i=1}^{\ell} \alpha_{i}(x) S(x) K_{i}^{\top} B(x)^{\top}|\Lambda(x)|^{-1}+\sum_{i=1}^{\ell} \alpha_{i}(x)|\Lambda(x)|^{-1} B(x) K_{i} S(x) \leq-2 \nu S(x)|\Lambda(x)|^{-1}
\end{aligned}
$$

and we let $K_{i}=U_{i} S^{-1}(0), i=1, \ldots, \ell$, which implies (36). This concludes the proof of Theorem 3.4.

Remark 3.5. The simultaneous synthesis of a boundary control and of a distributed control is possible. For the exponential kernel it consists in the computation of matrices $S^{-}, S^{+}, U$, and $U_{i}, i=1, \ldots, \ell$, satisfying (26), (28), and (36). For the affine kernel it consists in the computation of matrices $S^{-}, S^{+}, U$, and $U_{i}, i=1, \ldots, \ell$, satisfying (B.1), (B.3), and (B.9). The motivation for applying both controllers may be from an optimal point of view. Indeed, two controllers give more degrees of freedom for governing the state equation and thus it allows to drive the system to the origin with a better performance. It may be possible that a system is not stabilisable using boundary or distributed control but it can be stabilised by combining the two types of controllers.

\section{Overapproximation Techniques}

Conditions (10) and (21) involve the spatial variable $x \in[0,1]$. Hence, the number of LMI is infinite. Thus, some overapproximation may be introduced in order to numerically check it. Here, the term overapproximation means that the original constraints are embedded in a larger set having nice structural properties which may be exploited (see, for instance, [5]). In this section we aim at giving some practical techniques for the stability analysis and the control synthesis in the context of the exponential kernel (8) only. As for Section 3, the case of the affine kernel (19) is considered in Appendix B.

For each conditions given up to know we propose a result for their overapproximation. We distinguish two cases: spatially varying and non-spatially varying $F$ and $\Lambda$. For the clarity of the exposition we propose a summary table of the results at the end of the section.

\subsection{Non-Spatially Varying Case}

Let us suppose $F(x)=F, \Lambda(x)=\Lambda$. The main goal of this section is to provide a way to numerically verify conditions of Propositions 2.1 and 2.5 and of Theorems 3.1, B.1, 3.4, and B.2.

For fixed $\mu$ in $\mathbb{R}, Q^{-}$in $\mathbb{R}^{m \times m}$, and $Q^{+}$in $\mathbb{R}^{(n-m) \times(n-m)}$, we write

$$
Q_{i j}=\operatorname{diag}\left[e^{2 \mu i} Q^{-}, e^{-2 \mu j} Q^{+}\right], \quad i, j=0,1 .
$$

Lemma 4.1. For all $x \in[0,1], \mathcal{Q}(x)$ lies in the convex hull of $\left\{Q_{00}, Q_{01}, Q_{11}\right\}$ if $\mu>0$ and of $\left\{Q_{00}, Q_{10}, Q_{11}\right\}$ if $\mu<0$. 
Proof. We assume that $\mu>0$, the case $\mu<0$ is analogous. $\mathcal{Q}: x \mapsto \mathcal{Q}(x)$ is a parametrized curve in the $\left(Q^{-}, Q^{+}\right)$plane. We can express it as an explicit curve. We have $e^{2 \mu x} e^{-2 \mu x}=1$, thus the expression of the explicit curve is given by

$$
h(X)=\frac{1}{X}, \quad X \in \rho=\left[e^{-2 \mu}, 1\right] .
$$

This curve is convex on this interval. Then,

$$
\frac{1}{\alpha e^{-2 \mu}+(1-\alpha)} \leq \alpha g\left(e^{-2 \mu}\right)+(1-\alpha) g(1), \quad \alpha \in(0,1)
$$

where $g(X)=\left(\frac{1-e^{2 \mu}}{1-e^{-2 \mu}}\right) X+e^{2 \mu}+1$. When $X$ lies in $\rho, g(X)$ describes the straight line between $Q_{00}$ and $Q_{11}$. Hence, for $X \in \rho$ one has $h(X) \leq g(X)$. Thus, $\mathcal{Q}(x)$ lies in the convex hull of $\left\{Q_{00}, Q_{01}, Q_{11}\right\}$.

Remark 4.2. In the proof of Lemma 4.1 we do not consider the case $\mu=0$. Indeed, we get $\mathcal{Q}(x)=\operatorname{diag}\left[Q^{-}, Q^{+}\right]$. Thus, matrix inequality (10) is a "classical" linear matrix inequality which does not involve the spatial variable $x$.

Proposition 4.3. If there exist $\nu>0, \mu$ in $\mathbb{R} \backslash\{0\}$, and symmetric positive definite matrices $Q^{-}$in $\mathbb{R}^{m \times m}$ and $Q^{+}$in $\mathbb{R}^{(n-m) \times(n-m)}$ such that

$$
\begin{aligned}
& Q_{i j} \Lambda=\Lambda Q_{i j}, \\
& -2 \mu Q_{i j}+F^{\top}|\Lambda|^{-1} Q_{i j}+Q_{i j}|\Lambda|^{-1} F \leq-2 \nu|\Lambda|^{-1} Q_{i j},
\end{aligned}
$$

hold for all $(i, j) \in\{(0,0),(0,1),(1,1)\}$ if $\mu>0$ and for all $(i, j) \in\{(0,0),(1,0),(1,1)\}$ if $\mu<0$, together with

$$
\left[\begin{array}{cc}
I_{m} & 0_{m, n-m} \\
G_{+-} & G_{++}
\end{array}\right]^{\top} Q_{00} \tilde{I}_{n, m}\left[\begin{array}{cc}
I_{m} & 0_{m, n-m} \\
G_{+-} & G_{++}
\end{array}\right] \leq\left[\begin{array}{cc}
G_{--} & G_{-+} \\
0_{n-m, m} & I_{n-m}
\end{array}\right]^{\top} Q_{11} \tilde{I}_{n, m}\left[\begin{array}{cc}
G_{--} & G_{-+} \\
0_{n-m, m} & I_{n-m}
\end{array}\right],
$$

then conditions (9), (10), and (11) are satisfied for all $x \in[0,1]$.

Proof. We have that

- the inequality (43) corresponds to (11),

- by Lemma 4.1, the constraints given by the equality (9) and LMI (10) are embedded in the polytope built by the points $Q_{00}, Q_{01}$, and $Q_{11}$.

Thus, conditions (9), (10), and (11) are satisfied. The proof of Proposition 4.3 is complete.

Remark 4.4. The approximation with the exponential kernel (8) can be made tighter by increasing the number of points describing the polytope embbeding the constraints given by condition (9) and LMIs (10), (11). For instance, on Figure 1 there are 4 points: $Q_{11}, \tilde{Q}, \breve{Q}$, and $Q_{00}$. The impact of the number of points is explored numerically in the next section.

Conditions for the construction of boundary and distributed controller can be overapproximated in the same way as the conditions for stability. Let us suppose that $H(x)=H$ and $B(x)=B$, for all $x \in[0,1]$. For fixed $\mu$ in $\mathbb{R}, S^{-}$in $\mathbb{R}^{m \times m}$, and $S^{+}$in $\mathbb{R}^{(n-m) \times(n-m)}$, we write, for $i, j=0,1$,

$$
\begin{aligned}
& S_{i j}=\operatorname{diag}\left[e^{-2 \mu i} S^{-}, e^{2 \mu j} S^{+}\right], \\
& \breve{S}_{i j}=\operatorname{diag}\left[e^{-2 \mu i} S^{-}, e^{-2 \mu j} S^{+}\right],
\end{aligned}
$$

and

$$
I_{n, m}^{i j}=\operatorname{diag}\left[e^{-2 \mu i} I_{m}, e^{2 \mu j} I_{n-m}\right]
$$




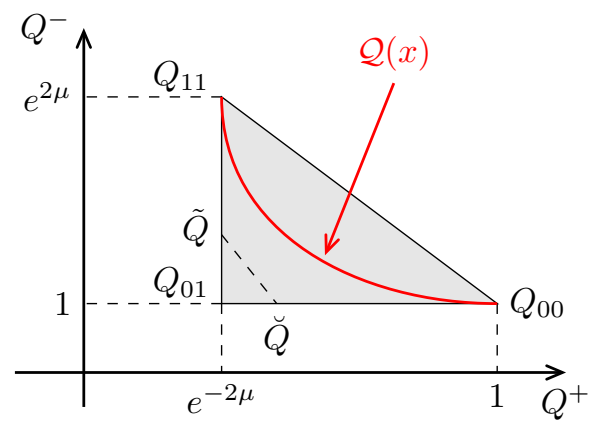

FigURE 1. Illustration of the elevation of number of points representing the polytope used for the overapproximation in the $\left(Q^{-}, Q^{+}\right)$-plane. Case $\mu>0$.

Proposition 4.5. If there exist $\nu>0, \mu$ in $\mathbb{R} \backslash\{0\}$, a matrix $U$ in $\mathbb{R}^{q \times n}$, and symmetric positive definite matrices $S^{-}$in $\mathbb{R}^{m \times m}, S^{+}$in $\mathbb{R}^{(n-m) \times(n-m)}$ such that the following conditions hold,

$$
\begin{aligned}
& S_{i j} \Lambda=\Lambda S_{i j}, \\
& -2 \mu S_{i j}+S_{i j} F^{\top}|\Lambda|^{-1}+|\Lambda|^{-1} F S_{i j} \leq-2 \nu S_{i j}|\Lambda|^{-1}, \\
& {\left[\begin{array}{cc}
\breve{S}_{01} & \left(T \breve{S}_{00}+L U\right)^{\top} \\
T \breve{S}_{00}+L U & \breve{S}_{10}
\end{array}\right] \geq 0,}
\end{aligned}
$$

for $(i, j) \in\{(0,0),(1,0),(1,1)\}$ if $\mu>0$ and for $(i, j) \in\{(0,0),(0,1),(1,1)\}$ if $\mu<0$, then conditions (26), (27), and (28) are satisfied for all $x \in[0,1]$.

Proof. We have that

- the inequality (28) corresponds to (49),

- by Lemma 4.1, the constraints given by the equality (26) and LMIs (27) are embedded in the polytope built by the points $S_{00}, S_{10}$, and $S_{11}$ if $\mu>0$ or by the points $S_{00}, S_{01}$, and $S_{11}$ if $\mu<0$.

Thus, conditions (26), (27), and (28) are satisfied. The proof of Proposition 4.5 is complete. 
Proposition 4.6. Let an integer $\ell>0$ be given. If there exist $\nu>0$, $\mu$ in $\mathbb{R} \backslash\{0\}$, matrices $U_{i}$ in $\mathbb{R}^{p \times n}$, $i=1, \ldots, \ell$, and symmetric positive definite matrices $S^{-}$in $\mathbb{R}^{m \times m}, S^{+}$in $\mathbb{R}^{(n-m) \times(n-m)}$ such that the following conditions hold,

$$
\begin{aligned}
& S_{j k} \Lambda=\Lambda S_{j k}, \\
& \left(|\Lambda|^{-1} H-\mu I_{n}\right) S_{j k}+S_{j k}\left(|\Lambda|^{-1} H-\mu I_{n}\right)^{\top} \\
& +\sum_{i=1}^{\ell} \min _{x \in[0,1]} \alpha_{i}(x)\left(I_{n, m}^{j k}\right)^{-1} U_{i}^{\top} B^{\top}|\Lambda|^{-1}+\sum_{i=1}^{\ell} \min _{x \in[0,1]} \alpha_{i}(x)|\Lambda|^{-1} B U_{i}\left(I_{n, m}^{j k}\right)^{-1} \leq-2 \nu S_{j k}|\Lambda|^{-1}, \\
& \left(|\Lambda|^{-1} H-\mu I_{n}\right) S_{j k}+S_{j k}\left(|\Lambda|^{-1} H(x)-\mu I_{n}\right)^{\top} \\
& +\sum_{i=1}^{\ell} \max _{x \in[0,1]} \alpha_{i}(x)\left(I_{n, m}^{j k}\right)^{-1} U_{i}^{\top} B^{\top}|\Lambda|^{-1}+\sum_{i=1}^{\ell} \max _{x \in[0,1]} \alpha_{i}(x)|\Lambda|^{-1} B U_{i}\left(I_{n, m}^{j k}\right)^{-1} \leq-2 \nu S_{j k}|\Lambda|^{-1}, \\
& {\left[\begin{array}{c}
\breve{S}_{01}\left(G \breve{S}_{00}\right) \\
\breve{S}_{00} \\
\breve{S}_{10}
\end{array}\right] \geq 0,}
\end{aligned}
$$

for $(j, k) \in\{(0,0),(1,0),(1,1)\}$ if $\mu>0$ and for $(j, k) \in\{(0,0),(0,1),(1,1)\}$ if $\mu<0$, then conditions (35), (36), and (37) are satisfied for all $x \in[0,1]$.

Proof. First of all the maximum and the minimum of $\alpha_{i}, i=1, \ldots, \ell$, are well-defined since $\alpha_{i}$ are continuous functions defined in the compact $[0,1]$. We have that

- the inequality (53) corresponds to (37),

- by Lemma 4.1, the constraints given by the equality (35) and LMI (36) are embedded in the polytope built by the points $S_{00}, S_{10}$, and $S_{11}$ if $\mu>0$ or by the points $S_{00}, S_{01}$, and $S_{11}$ if $\mu<0$. Noting that any value of a continuous function can be expressed as a convex combination of its maximum and minimum, condition (36) holds for all $x \in[0,1]$.

The proof of Proposition 4.6 is complete.

\subsection{Spatially-Varying Case}

We may generalize the previous results when $\Lambda$ and $F$ are both spatially varying and lie in a convex hull.

We assume that the parametrized matrix

$$
W(x)=|\Lambda(x)|^{-1} F(x)
$$

lies for all $x \in[0,1]$ in the convex hull

$$
\mathcal{W}:=\left\{W: W=\sum_{i=1}^{N} \alpha_{i} W_{i}, \sum_{i=1}^{N} \alpha_{i}=1\right\}
$$

for given matrices $W_{i}, i=1, \ldots, N$. We have the following propositions.

Proposition 4.7. If there exist $\nu>0, \mu$ in $\mathbb{R} \backslash\{0\}$, and diagonal positive definite matrices $Q^{-}$in $\mathbb{R}^{m \times m}$ and $Q^{+}$in $\mathbb{R}^{(n-m) \times(n-m)}$ such that

$$
\begin{aligned}
& -2 \mu Q_{j k}+W_{i}^{\top} Q_{j k}+Q_{j k} W_{i} \leq-2 \nu|\bar{\Lambda}|^{-1} Q_{j k}, \\
& {\left[\begin{array}{cc}
I_{m} & 0_{m, n-m} \\
G_{+-} & G_{++}
\end{array}\right]^{\top} \tilde{I}_{n, m} Q_{00}\left[\begin{array}{cc}
I_{m} & 0_{m_{n, n-m}} \\
G_{+-} & G_{++}
\end{array}\right] \leq\left[\begin{array}{cc}
G_{--} & G_{-+} \\
0_{n-m, m} & I_{n-m}
\end{array}\right]^{\top} \tilde{I}_{n, m} Q_{11}\left[\begin{array}{cc}
G_{--} & G_{-+} \\
0_{n-m, m} & I_{n-m}
\end{array}\right],}
\end{aligned}
$$


where

$$
\bar{\Lambda}=\operatorname{diag}\left[\min _{x \in[0,1]} \lambda_{1}(x), \ldots, \min _{x \in[0,1]} \lambda_{m}(x), \max _{x \in[0,1]} \lambda_{m+1}(x), \ldots, \max _{x \in[0,1]} \lambda_{n}(x)\right]
$$

for all $i=1, \ldots, N$, and $(j, k) \in\{(0,0),(0,1),(1,1)\}$ if $\mu>0,(j, k) \in\{(0,0),(1,0),(1,1)\}$ if $\mu<0$, then conditions (9), (10), and (11) are satisfied for all $x \in[0,1]$.

Proof. Multiplying (56) by $\alpha_{i}$ and making the sum for $i=1, \ldots, N$, we get

$$
-2 \mu Q_{j k}+W(x)^{\top} Q_{j k}+Q_{j k} W(x) \leq-2 \nu|\bar{\Lambda}|^{-1} Q_{j k},
$$

for all $x \in[0,1],(j, k)=\{(0,0),(0,1),(1,1)\}$ if $\mu>0$ and $(j, k)=\{(0,0),(1,0),(1,1)\}$ if $\mu<0$.

- Using Lemma 4.1 and the definition of $\bar{\Lambda}$ in (58), one gets (10).

- Condition (9) is automatically satisfied because of the diagonal form of $Q^{-}$and $Q^{+}$.

The proof of Proposition 4.7 is complete.

Remark 4.8. In the statement of Proposition 4.7 we assume that the matrices $Q^{-}$and $Q^{+}$are diagonal. It allows to avoid to add an other assumption on the structure of $\Lambda$. For the results related to the spatially-varying case we will always state this assumption.

Proposition 4.9. If there exist $\nu>0, \mu$ in $\mathbb{R} \backslash\{0\}$, a matrix $U$ in $\mathbb{R}^{q \times n}$, and diagonal positive definite matrices $S^{-}$in $\mathbb{R}^{m \times m}, S^{+}$in $\mathbb{R}^{(n-m) \times(n-m)}$ such that the following conditions hold,

$$
\begin{aligned}
& -2 \mu S_{j k}+S_{j k} W_{i}^{\top}+W_{i} S_{j k} \leq-2 \nu S_{j k}|\bar{\Lambda}|^{-1}, \\
& {\left[\begin{array}{cc}
\breve{S}_{01} & \left(T \breve{S}_{00}+L U\right)^{\top} \\
T \breve{S}_{00}+L U & \breve{S}_{10}
\end{array}\right] \geq 0,}
\end{aligned}
$$

for all $i=1, \ldots, N$, and $(j, k) \in\{(0,0),(1,0),(1,1)\}$ if $\mu>0,(j, k) \in\{(0,0),(0,1),(1,1)\}$ if $\mu<0$, then conditions (26), (27), and (28) are satisfied for all $x \in[0,1]$.

Proof. As in the proof of Proposition 4.7 we multiply (60) by $\alpha_{i}$, and make the sum for $i=1, \ldots, N$, getting

$$
-2 \mu S_{j k}+S_{j k} W(x)^{\top}+W(x) S_{j k} \leq-2 \nu S_{j k}|\bar{\Lambda}|^{-1},
$$

for all $x \in[0,1],(j, k) \in\{(0,0),(1,0),(1,1)\}$ if $\mu>0,(j, k) \in\{(0,0),(0,1),(1,1)\}$ if $\mu<0$.

- Using Lemma 4.1 and the definition of $\bar{\Lambda}$ in (58), one gets (27).

- Using the diagonal form of $S^{-}$and $S^{+}$, condition (26) is automatically satisfied.

- Condition (61) is equivalent to condition (28).

The proof of Proposition 4.9 is complete.

For the distributed controller synthesis, in the case where $H$ as well as $B$ are spatially-varying we may generalize the above method. We assume that the parametrized matrices

$$
|\Lambda(x)|^{-1} H(x),
$$

and

lie, respectively, in

$$
|\Lambda(x)|^{-1} B(x),
$$

$$
\mathcal{R}:=\left\{R: R=\sum_{i=1}^{M_{1}} \beta_{i} R_{i}, \sum_{i=1}^{M_{1}} \beta_{i}=1\right\},
$$


and

$$
\mathcal{Z}:=\left\{Z: Z=\sum_{i=1}^{M_{2}} \gamma_{i} Z_{i}, \sum_{i=1}^{M_{2}} \gamma_{i}=1\right\}
$$

for given matrices $R_{i}, Z_{j}, i=1, \ldots, M_{1}, j=1, \ldots, M_{2}$. In this context we have the following propositions.

Proposition 4.10. Let an integer $\ell>0$ be given. If there exist $\nu>0, \mu$ in $\mathbb{R} \backslash\{0\}$, matrices $U_{i}$ in $\mathbb{R}^{p \times n}$, $i=1, \ldots, \ell$, and diagonal positive definite matrices $S^{-}$in $\mathbb{R}^{m \times m}, S^{+}$in $\mathbb{R}^{(n-m) \times(n-m)}$ such that the following conditions hold

$$
\begin{aligned}
& \left(R_{\iota_{1}}-\mu I_{n}\right) S_{j k}+S_{j k}\left(R_{\iota_{1}}-\mu I_{n}\right)^{\top} \\
& +\sum_{i=1}^{\ell} \min _{x \in[0,1]} \alpha_{i}(x)\left(I_{n, m}^{j k}\right)^{-1} U_{i}^{\top} Z_{\iota_{2}}^{\top}+\sum_{i=1}^{\ell} \min _{x \in[0,1]} \alpha_{i}(x) Z_{\iota_{2}} U_{i}\left(I_{n, m}^{j k}\right)^{-1} \leq-2 \nu S_{j k}|\bar{\Lambda}|^{-1} \\
& \left(R_{\iota_{1}}-\mu I_{n}\right) S_{j k}+S_{j k}\left(R_{\iota_{1}}-\mu I_{n}\right)^{\top} \\
& +\sum_{i=1}^{\ell} \max _{x \in[0,1]} \alpha_{i}(x)\left(I_{n, m}^{j k}\right)^{-1} U_{i}^{\top} Z_{\iota_{2}}^{\top}+\sum_{i=1}^{\ell} \max _{x \in[0,1]} \alpha_{i}(x) Z_{\iota_{2}} U_{i}\left(I_{n, m}^{j k}\right)^{-1} \leq-2 \nu S_{j k}|\bar{\Lambda}|^{-1}, \\
& {\left[\begin{array}{c}
\breve{S}_{01}\left(G \breve{S}_{00}\right)^{\top} \\
G \breve{S}_{00} \breve{S}_{10}
\end{array}\right] \geq 0,}
\end{aligned}
$$

for all $\iota_{1}=1, \ldots, M_{1}, \iota_{2}=1, \ldots, M_{2}$, and $(j, k) \in\{(0,0),(1,0),(1,1)\}$ if $\mu>0,(j, k) \in\{(0,0),(0,1),(1,1)\}$ if $\mu<0$, then conditions (35), (36), and (37) are satisfied for all $x \in[0,1]$.

Proof. We multiply (67) and $(68)$ by $\beta_{\iota_{1}}, \gamma_{\iota_{2}}$, and make the sum for $\iota_{1}=1, \ldots, M_{1}, \iota_{2}=1, \ldots, M_{2}$, we get

$$
\begin{aligned}
& \left(R(x)-\mu I_{n}\right) S_{j k}+S_{j k}\left(R(x)-\mu I_{n}\right)^{\top} \\
& +\sum_{i=1}^{\ell} \min _{x \in[0,1]} \alpha_{i}(x)\left(I_{n, m}^{j k}\right)^{-1} U_{i}^{\top} Z(x)^{\top}+\sum_{i=1}^{\ell} \min _{x \in[0,1]} \alpha_{i}(x) Z(x) U_{i}\left(I_{n, m}^{j k}\right)^{-1} \leq-2 \nu|\bar{\Lambda}|^{-1} S_{j k}, \\
& \left(R(x)-\mu I_{n}\right) S_{j k}+S_{j k}\left(R(x)-\mu I_{n}\right)^{\top} \\
& +\sum_{i=1}^{\ell} \max _{x \in[0,1]} \alpha_{i}(x)\left(I_{n, m}^{j k}\right)^{-1} U_{i}^{\top} Z(x)^{\top}+\sum_{i=1}^{\ell} \max _{x \in[0,1]} \alpha_{i}(x) Z(x) U_{i}\left(I_{n, m}^{j k}\right)^{-1} \leq-2 \nu|\bar{\Lambda}|^{-1} S_{j k} .
\end{aligned}
$$

- Using Lemma 4.1, the definition of $\bar{\Lambda}$ in (58) and the fact that any value of a continuous function can be expressed as a convex combination of its minimum and maximum, one gets (36).

- Condition (35) is automatically satisfied because of the diagonal form of $S^{-}$and $S^{+}$.

- Condition (69) is equivalent to condition (37).

The proof of Proposition 4.10 is complete. 
Since Section 4 gathers a lot of results we propose a summary table.

\begin{tabular}{|l|l|l|l|}
\hline \multicolumn{2}{|c|}{ Stability Analysis } & Non Spatially Varying & Proposition 4.3 \\
\cline { 3 - 4 } & & Spatially Varying & Proposition 4.7 \\
\hline \multirow{4}{*}{ Controller Synthesis } & \multirow{2}{*}{ Boundary Control } & Non Spatially Varying & Proposition 4.5 \\
\cline { 3 - 4 } & & Spatially Varying & Proposition 4.9 \\
\cline { 2 - 4 } & \multirow{2}{*}{ Distributed Control } & Non Spatially Varying & Proposition 4.6 \\
\cline { 3 - 4 } & & Spatially Varying & Proposition 4.10 \\
\hline
\end{tabular}

TABLE 1. Summary table of the results of Section 4 relative to the exponential kernel (8).

\section{Numerical Experiments}

In this section, several examples are presented to illustrate the results of the paper. All the solutions of the LMIs have been computed with the Yalmip solver [14].

\subsection{Stability Analysis, illustrating Proposition 4.3}

Example 5.1. Let us consider the following matrices

$$
\begin{aligned}
\Lambda & =\operatorname{diag}[-3,1], \\
F & =\left[\begin{array}{cc}
-1 & 0.2 \\
1 & 0.2
\end{array}\right], \\
G & =\left[\begin{array}{cc}
0.2 & -0.3 \\
0.6 & 0.1
\end{array}\right] .
\end{aligned}
$$

The matrix $F$ in $(72)$ is non-Hurwitz and the matrix $G$ in $(73)$ is such that $\rho(G)<1$. This last property is classical for the stability analysis of linear and quasilinear hyperbolic system [6], [10].

Figure 2 shows that the result obtained with only three points for the polytope is optimal. Indeed the numerical value of $\nu$ obtained with three points is the same than with higher number of points. This result might be expected because all the constraints of the LMI are enclosed by the overapproximation with the polytope described by three points. The lower curve corresponds to the result of the algorithm when the objective is to maximize $\nu$. In order to make this objective tractable, a relaxation on the right-hand side of the inequality (10) is made. The upper curve is the result of the algorithm when the objective is to minimize the trace of $\mathcal{Q}(0)$. Unexpectedly, the second algorithm gives a better $\nu$ than the first one.

The Lyapunov function (7) with the affine kernel (19) does not converge, which is not surprising when we look at LMIs (B.16) and (B.17) in Appendix B. Indeed, to satisfy these latter LMIs we need a large positive $\mu$, that means a $\mu$ near to be one, but in the same time increasing the $\mu$ will make the verification of (B.18) complex. Hence, the Lyapunov function (7) with the affine kernel (19) may not give results when the matrix $F$ is not Hurwitz.

Example 5.2. Let us consider the following matrices

$$
\begin{aligned}
\Lambda & =\operatorname{diag}[-1,1], \\
F & =\left[\begin{array}{cc}
-0.3 & 0.1 \\
0.1 & -0.3
\end{array}\right], \\
G & =\left[\begin{array}{ll}
0.1 & -0.8 \\
0.6 & -0.4
\end{array}\right] .
\end{aligned}
$$

In this example the matrix $F$ in $(74)$ is Hurwitz and the matrix $G$ in $(75)$ is contractive that is $\rho(G)<1$. 


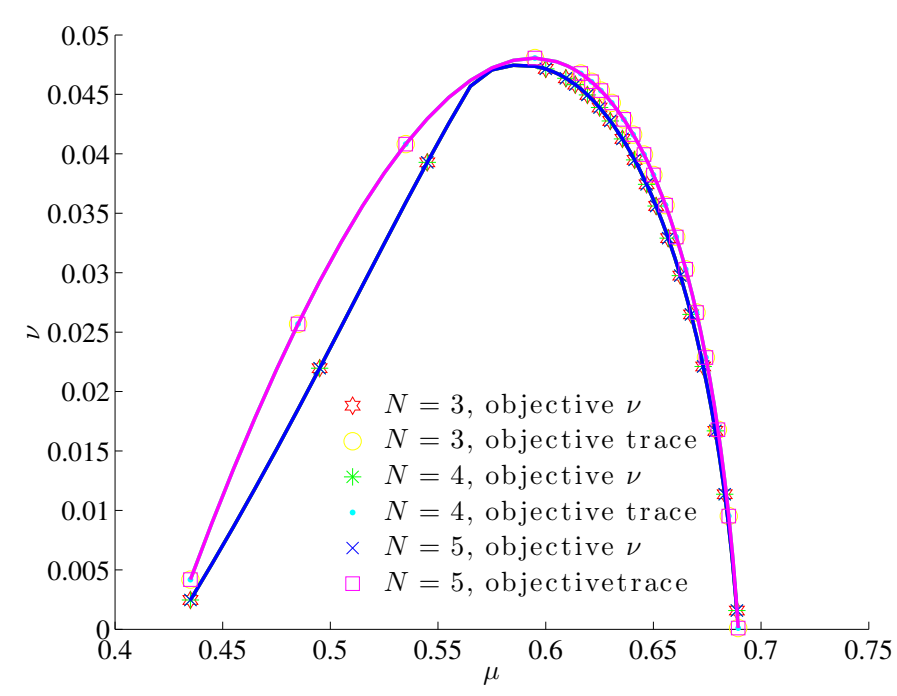

Figure 2. Evolution of $\nu$ as a function of $\mu$ for Example 5.1 for the gridding method depending on the number of points.

Figure 3 shows that the algorithm for which the objective is to minimize the trace of $\mathcal{Q}(0)$ seems to give a better $\nu$ than the algorithm for which the objective is to maximize $\nu$. Moreover, it shows that the Lyapunov function (7) with the affine kernel (19) gives a better $\nu$ for the first $\mu$ than the exponential kernel (8), and gives solutions at some $\mu$ while the other kernel fails. Moreover, the shape of the curve obtained in this example is not the same as the one presented in Example 5.1. This comes from the fact that the matrix $F$ is Hurwitz, hence increasing $\mu$ moves the eigenvalues of $|\Lambda|^{-1} F-\mu I_{2}$ in the left half-plane of $\mathbb{C}$, so it will increase the parameter $\nu$. The algorithm stops due to LMI (11) which is no more solvable for large $\mu$. Thus, this example illustrates also Proposition 2.3.

\subsection{Controller Design, illustrating Theorems 3.1, B.1, and 3.4}

Example 5.3. Let us consider system (1)-(3) with

$$
\begin{aligned}
& \Lambda=\operatorname{diag}[-1,2], \\
& F=\left[\begin{array}{cc}
-0.1 & 0.1 \\
0.5 & -0.8
\end{array}\right],
\end{aligned}
$$

under the boundary control (25) where

$$
\begin{aligned}
T & =\left[\begin{array}{cc}
-0.5 & 1 \\
0.5 & 1
\end{array}\right], \\
L^{\top} & =\left[\begin{array}{ll}
0.5 & -1
\end{array}\right] .
\end{aligned}
$$

Let us choose $\nu=0.1$. The design algorithm gives

$$
\begin{aligned}
\mu & =0.1580, \\
K_{B} & =\left[\begin{array}{ll}
0.5596 & 0.7910
\end{array}\right],
\end{aligned}
$$




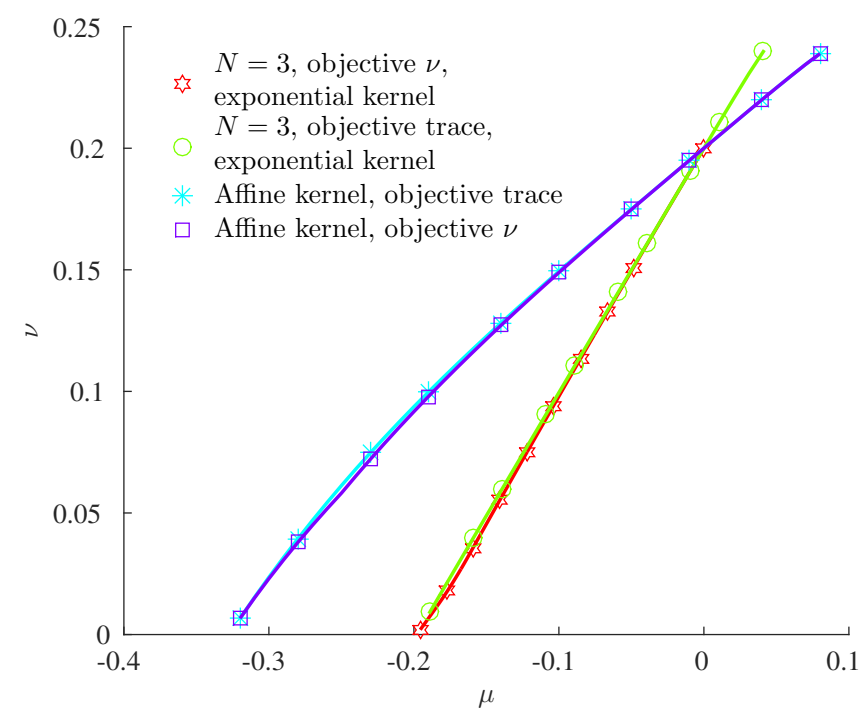

Figure 3. Evolution of $\nu$ as a function of $\mu$ for Example 5.2.

which lead to the following

$$
G=\left[\begin{array}{ll}
-0.2202 & 1.3955 \\
-0.0596 & 0.2090
\end{array}\right]
$$

We check numerically the behavior of the solution to (1)-(3) with the matrix $G$ given by (78), with a two-step variant of the Lax-Friedrichs $(\mathrm{LxF})$ method [16]. The initial condition is chosen as

$$
y^{0}(x)=\left[\begin{array}{c}
\sqrt{2} \sin (\pi x) \\
\sqrt{2} \sin (2 \pi x)
\end{array}\right], \quad x \in[0,1] .
$$

Figure 4 shows the evolution of the state of the system (1)-(3) with initial condition given by (79) and under the boundary control (78).

Example 5.4. Let us consider system (1)-(3) with

$$
\begin{aligned}
\Lambda & =\operatorname{diag}[-2,1], \\
G & =\left[\begin{array}{cc}
0.5 & -0.4 \\
0.2 & 0.8
\end{array}\right],
\end{aligned}
$$

under the distributed control (32) where

$$
\begin{aligned}
H & =\left[\begin{array}{cc}
-0.5 & 0.2 \\
0.2 & 0.5
\end{array}\right], \\
B^{\top} & =\left[\begin{array}{ll}
0.5 & 1
\end{array}\right], \\
(\ell, \alpha) & =(1,1) .
\end{aligned}
$$

Numerically, $\nu$ is fixed to 0.3 . The design algorithm gives

$$
\begin{aligned}
\mu & =0.15, \\
K_{D} & =\left[\begin{array}{ll}
-0.3130 & -1.1485
\end{array}\right],
\end{aligned}
$$



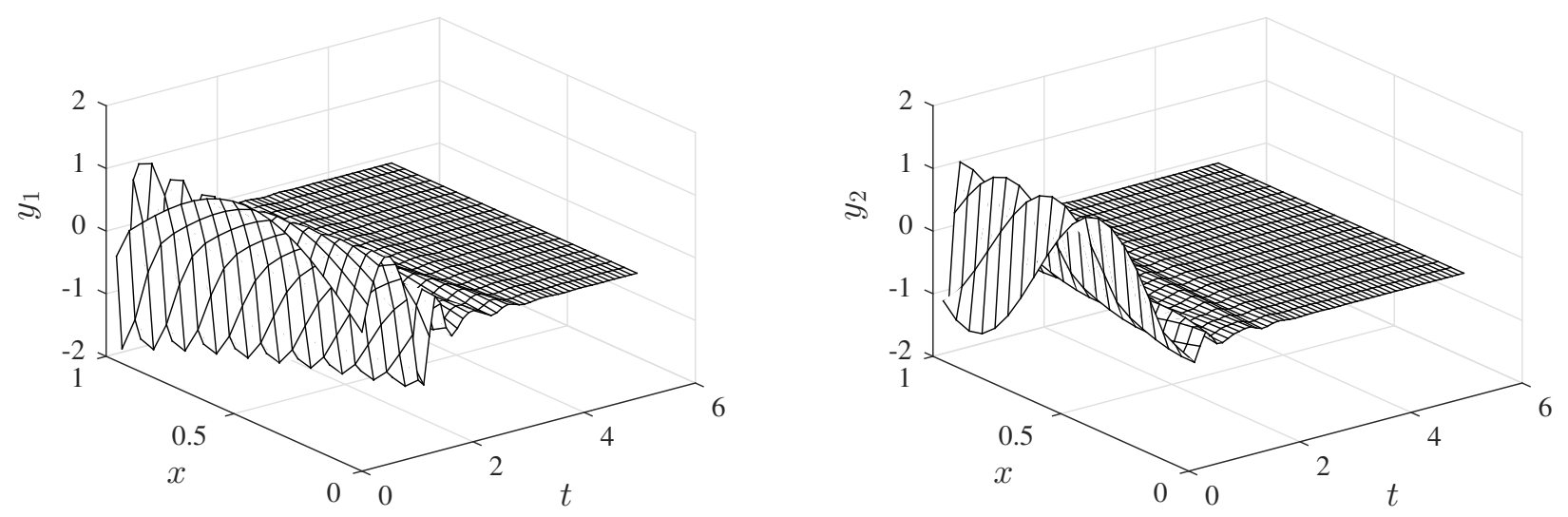

Figure 4. Evolution of the first component $y_{1}$ (left) and of the second component $y_{2}$ (right) of system (1)-(3) with $\Lambda$ and $F$ given, respectively, by (76) and (77), initial condition given by (79), and under the boundary control (78).
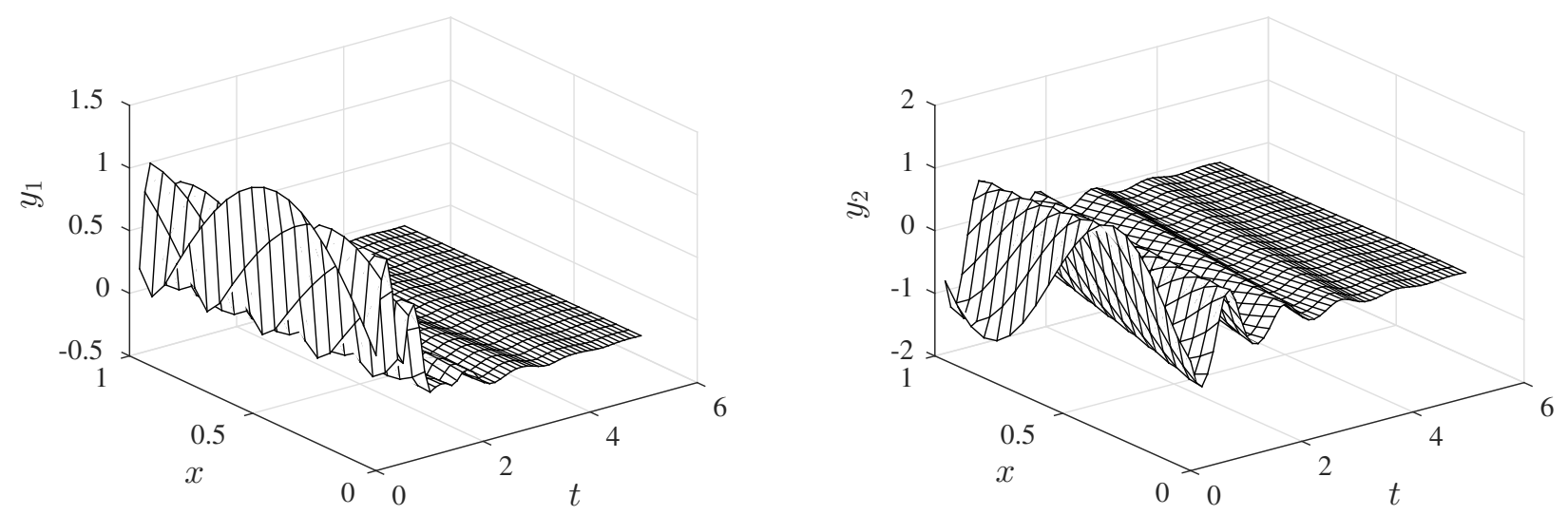

Figure 5. Evolution of the first component $y_{1}$ (left) and of the second component $y_{2}$ (right) of system (1)-(3) with $\Lambda$ and $G$ given, respectively, by (80) and (81), initial condition given by (79), and under the distributed control (82).

which lead to the following

$$
F=\left[\begin{array}{ll}
-0.6565 & -0.3743 \\
-0.1130 & -0.6485
\end{array}\right]
$$

Figure 5 shows the evolution of the state of the system (1)-(3) with initial condition given by (79) and under the distributed control (82). 


\subsection{Saint-Venant Equations}

We illustrate Proposition 4.7 with the Saint-Venant equations for a prismatic horizontal open channel with a rectangular cross-section and a unit width, as considered in [1]. The dynamics driven the water velocity $V(t, x)$ and the water level $H(t, x)$ in the pool at time $t \in \mathbb{R}^{+}$and space-location $x \in[0, L]$, are described by the Saint-Venant equations

$$
\begin{aligned}
\partial_{t} H(t, x)+\partial_{x}(H(t, x) V(t, x)) & =0, \\
\partial_{t} V(t, x)+\partial_{x}\left(\frac{V^{2}}{2}(t, x)+g H(t, x)\right)+g C \frac{V^{2}(t, x)}{H(t, x)} & =0,
\end{aligned}
$$

with $C$ a friction coefficient and $g$ the gravity acceleration. The channel is provided with some control devices allowing to assign the flow-rate on both sides of the channel, that is

$$
\begin{aligned}
& Q_{1}(t)=H(t, 0) V(t, 0), \\
& Q_{2}(t)=H(t, L) V(t, L) .
\end{aligned}
$$

System (83), (84) is non-linear, and the objective is to stabilize the water level at a desired steady state $\left(H^{*}, V^{*}\right)$

which is constant with respect to time but depends on the space variable. Hence, system (83), (84) is linearized around the steady state $\left(H^{*}, V^{*}\right)$, and write in characteristic coordinates. We get

$$
\begin{aligned}
& \partial_{t} y_{1}(t, x)-\lambda_{1}(x) \partial_{x} y_{1}(t, x)=\gamma_{1}(x) y_{1}(t, x)+\delta_{1}(x) y_{2}(t, x), \\
& \partial_{t} y_{2}(t, x)+\lambda_{2}(x) \partial_{x} y_{2}(t, x)=\gamma_{2}(x) y_{1}(t, x)+\delta_{2}(x) y_{2}(t, x),
\end{aligned}
$$

with the characteristics velocities

$$
\begin{aligned}
-\lambda_{1}(x) & =V^{*}(x)-\sqrt{g H^{*}(x)}, \\
\lambda_{2}(x) & =V^{*}(x)+\sqrt{g H^{*}(x)},
\end{aligned}
$$

and the spatially-varying coefficients

$$
\begin{aligned}
& \gamma_{1}(x)=\frac{g C V^{*^{2}}(x)}{H^{*}(x)}\left[\frac{3}{4\left(\sqrt{g H^{*}(x)}+V^{*}(x)\right)}-\frac{1}{V^{*}(x)}+\frac{1}{2 \sqrt{g H^{*}(x)}}\right]+\frac{3 g S_{b}}{4\left(\sqrt{g H^{*}(x)}+V^{*}(x)\right)}, \\
& \gamma_{2}(x)=\frac{g C V^{*^{2}}(x)}{H^{*}(x)}\left[\frac{3}{4\left(\sqrt{g H^{*}(x)}-V^{*}(x)\right)}-\frac{1}{V^{*}(x)}+\frac{1}{2 \sqrt{g H^{*}(x)}}\right]+\frac{g S_{b}}{4\left(\sqrt{g H^{*}(x)}+V^{*}(x)\right)}, \\
& \delta_{1}(x)=\frac{g C V^{*^{2}}(x)}{H^{*}(x)}\left[\frac{3}{4\left(\sqrt{g H^{*}(x)}+V^{*}(x)\right)}-\frac{1}{V^{*}(x)}-\frac{1}{2 \sqrt{g H^{*}(x)}}\right]-\frac{g S_{b}}{4\left(\sqrt{g H^{*}(x)}+V^{*}(x)\right)}, \\
& \delta_{2}(x)=\frac{g C V^{*^{2}}(x)}{H^{*}(x)}\left[\frac{3}{4\left(\sqrt{g H^{*}(x)}-V^{*}(x)\right)}-\frac{1}{V^{*}(x)}-\frac{1}{2 \sqrt{g H^{*}(x)}}\right]-\frac{3 g S_{b}}{4\left(\sqrt{g H^{*}(x)}+V^{*}(x)\right)} .
\end{aligned}
$$

The steady state $\left(H^{*}, V^{*}\right)$ satisfies $H^{*}(x) V^{*}(x)=Q^{*}$ where $Q^{*}$ is a constant and $V^{*}$ satisfies the ODE

$$
\frac{d V^{*}(x)}{d x}=\frac{g C}{Q^{*}(x)}\left[\frac{V^{*^{5}}(x)}{g Q^{*}(x)-V^{*^{3}}(x)}\right], \quad x \in[0, L],
$$


with the initial condition

$$
V^{*}(0)=V_{0}^{*} .
$$

The numerical values chosen are $L=1 \mathrm{~km}, g=9.81 \mathrm{~m} \cdot \mathrm{s}^{-2}, C=0.002 \mathrm{~s}^{2} \cdot \mathrm{m}^{-1}, Q^{*}=H^{*} V^{*}=1 \mathrm{~m}^{3} \cdot \mathrm{s}^{-1}$, and the initial condition $V_{0}^{*}=0.5 \mathrm{~m} . \mathrm{s}^{-1}$. We compute numerically the matrices $W_{i}$ for

$$
\mu=1.4
$$

We compute numerically the steady state and the polytope $\mathcal{W}$ given by $W \in \mathcal{W}$ if and only if $W_{i, j} \in\left[\min _{x \in[0,1]} W_{i, j}(x), \max _{x \in[0,1]} W_{i, j}(x)\right]$. This corresponds to the convex hull of 16 matrices $W_{i}$. Let us assume the controllers (85) and (86) are such that in the characteristic coordinates one has

$$
\left[\begin{array}{l}
y_{1}(t, 1) \\
y_{2}(t, 0)
\end{array}\right]=\left[\begin{array}{cc}
0 & 0.2 \\
0.3 & 0
\end{array}\right]\left[\begin{array}{l}
y_{1}(t, 0) \\
y_{2}(t, 1)
\end{array}\right]
$$

It must be stressed that all of this values may be prone to numerical errors since the explicit expression of $V^{*}$ is unknown. Next, we compute matrices $Q^{-}$and $Q^{+}$such that Proposition 4.7 holds. Conditions (56)-(57) are checked with

$$
\begin{aligned}
Q^{-} & =1, \\
Q^{+} & =10.8171, \\
\nu & =4.8432
\end{aligned}
$$

Thus, this numerical method is effective on physical device. Figure 6 shows the evolution of the Lyapunov function $V$ given by (7) with the kernel (8) where $Q^{-}, Q^{+}$, and $\mu$ are given by (91), (92), and (89) respectively, along the numerical solution to system (87), (88) with boundary condition (90), and of the theoretical bound $e^{-2 \nu t} V\left(y^{0}\right)$ where $\nu$ and $y^{0}$ are given by (93) and (79) respectively. The theoretical bound $\nu$ obtained numerically is close to the exponential decay of the solution obtained by the numerical integration of the characteristic Saint-Venant equations (87), (88), and (90).

\section{Conclusion}

In this work, the stability of a hyperbolic system over a bounded interval with spatially-varying coefficients was analysed. We have proposed a Lyapunov function and stability conditions based on its time-derivative along the solutions to the system. These conditions are expressed as LMIs. We also design boundary and distributed controllers by the same techniques. All these conditions correspond to an infinite number of LMIs. Hence, we proved that this numerical complexity can be relaxed. We show the effectiveness of the method with academic examples and with the Saint-Venant equations with friction.

\section{REFERENCES}

[1] G. Bastin and J.-M. Coron. On boundary feedback stabilization of non-uniform linear hyperbolic systems over a bounded interval. Systems \& Control Letters, 60:900-906, 2011.

[2] G. Bastin and J.-M. Coron. Stability and Boundary Stabilization of 1-D Hyperbolic Systems. PNLDE Subseries in Control. Springer, 2016.

[3] S. Boyd, L. El Ghaoui, E. Feron, and V. Balakrishnan. Linear Matrix Inequalities in System and Control Theory. Society for industrial and applied mathematics, Philadelphia, 1994.

[4] F. Castillo, E. Witrant, C. Prieur, and L. Dugard. Boundary observers for linear and quasi-linear hyperbolic systems with application to flow control. Automatica, 49:3180-3188, 2013.

[5] F. Castillo, E. Witrant, C. Prieur, V. Talon, and L. Dugard. Fresh air fraction control in engines using dynamic boundary stabilization of LPV hyperbolic systems. IEEE Transactions on Control Systems Technology, 23(3):963-974, 2015. 


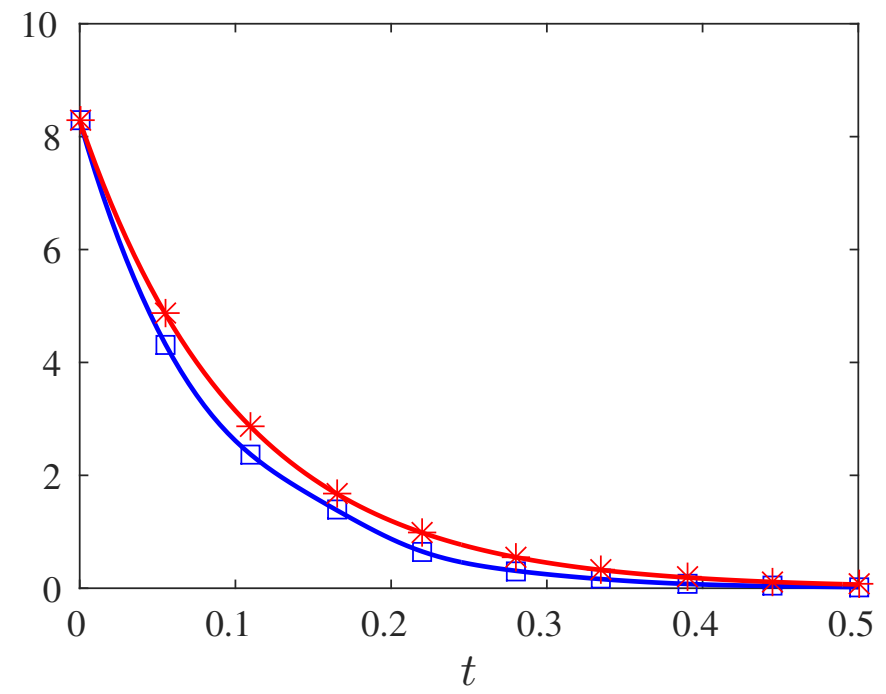

Figure 6. Evolution of the Lyapunov function $V$ given by (7) with the kernel (8) where $Q^{-}, Q^{+}$, and $\mu$ are given by (91), (92), and (89) respectively, along the numerical solution to system (87), (88) with boundary condition (90) (square marker), and of the theoretical bound $e^{-2 \nu t} V\left(y^{0}\right)$ where $\nu$ and $y^{0}$ are given by (93) and (79) respectively (star marker).

[6] J.-M. Coron, G. Bastin, and B. d'Andréa Novel. Dissipative boundary conditions for one dimensional nonlinear hyperbolic systems. SIAM Journal on Control and Optimization, 47:1460-1498, 2008.

[7] J.-M. Coron, B. d'Andréa Novel, and G. Bastin. A strict Lyapunov function for boundary control of hyperbolic systems of conservation laws. IEEE Transactions on Automatic Control, 52:2-11, 2007.

[8] J. Daafouz, M. Tucsnak, and J. Valein. Nonlinear control of a coupled PDE/ODE system modeling a switched power converter with a transmission line. Systems $\&$ Control Letters, 70:92-99, 2014.

[9] F. Di Meglio, R. Vazquez, and M. Krstic. Stabilization of a system of $n+1$ coupled first-order hyperbolic linear PDEs with a single boundary input. IEEE Transactions on Automatic Control, 58(12):3097-3111, 2013.

[10] A. Diagne, G. Bastin, and J.-M. Coron. Lyapunov exponential stability of 1-D linear hyperbolic systems of balance laws. Automatica, 48:109-114, 2012.

[11] E. Fridman and Y. Orlov. An LMI approach to $H^{\infty}$ boundary control of semilinear parabolic and hyperbolic systems. Automatica, 45:2060-2066, 2009.

[12] I. Karafyllis and M. Krstic. On the relation of delay equations to first-order hyperbolic partial differential equations. ESAIM: Control, Optimisation and Calculus of Variations, 20(3):894-923, 2014.

[13] I. Karafyllis, M. Malisoff, and M. Krstic. Ergodic theorem for stabilization of a hyperbolic PDE inspired by age-structured chemostat. Preprint submitted to arxiv on January 2015. Available at: arxiv.org/pdf/1501.04321.pdf.

[14] J. Löfberg. YALMIP: A toolbox for modeling and optimization in MATLAB. In IEEE International Symposium on Computer Aided Control Systems Design, 2004.

[15] C. Prieur, A. Girard, and E. Witrant. Stability of switched linear hyperbolic systems by Lyapunov techniques. IEEE Transactions on Automatic Control, 59:2196-2202, 2014

[16] L. F. Shampine. Solving hyperbolic PDEs in MATLAB. Applied Numerical Analysis \& Computational Mathematics, 2:346-358, 2005.

[17] C. Z. Xu and G. Sallet. Exponential stability and transfer functions of processes governed by symmetric hyperbolic systems. ESAIM: Control, Optimisation and Calculus of Variations, 7:421-442, 2002. 


\section{Appendix A. Proof about Remark 2.6}

Let us prove that there do not exist a real $\mu$ and a diagonal matrix $Q$ such that conditions (10) and (11) hold for the example of Remark 2.6.

Condition (10) for stability is rewritten as

$$
\left(F-\mu I_{2}\right)^{\top} Q+Q\left(F-\mu I_{2}\right)<0
$$

Condition (11) is rewritten as

$$
\frac{1}{4} Q \leq e^{-2 \mu} Q
$$

which implies $\frac{1}{4} \leq e^{-2 \mu}$ that is $\mu \leq \frac{\ln (4)}{2}=\lambda$. To find a matrix $F$ such that it does not exist a diagonal $Q$ such that (A.1) hold, we suppose that $F$ is written as

$$
F=A+\lambda I_{2}=\left[\begin{array}{cc}
a+\lambda & b \\
c & d+\lambda
\end{array}\right]
$$

where $A=\left[\begin{array}{ll}a & b \\ c & d\end{array}\right]$. Using (A.1) the matrix $A$ shall satisfy

$$
A^{\top} Q+Q A<2(\mu-\lambda) Q \leq 0
$$

To insure the existence of a solution to the latter Lyapunov equation we suppose that $A$ is Hurwitz $(\operatorname{trace}(A)=$ $a+d<0, \operatorname{det}(A)=a d-b c>0)$. Without loss of generality the diagonal matrix $Q$ can be written as $\left[\begin{array}{ll}1 & 0 \\ 0 & \alpha\end{array}\right]$, with $\alpha>0$. One gets

$$
Q A+A^{\top} Q=\left[\begin{array}{cc}
2 a & b+\alpha c \\
b+\alpha c & 2 \alpha d
\end{array}\right]
$$

The determinant of the above matrix is given by $4 a d \alpha-(b+\alpha c)^{2}$, which is a polynomial of degree 2 in $\alpha$,

$$
P(\alpha)=-\alpha^{2} c^{2}+(4 a d-2 b c) \alpha-b^{2} .
$$

The objective is to obtain a matrix $A$ such that the Lyapunov inequality $A^{\top} Q+Q A<0$ has no diagonal solution. Hence, if the determinant is negative the counter example is obtained. The determinant of $P$ satisfies

$$
\begin{aligned}
(4 a d-2 b c)^{2}-4 b^{2} c^{2}<0 & \Leftrightarrow 16 a^{2} d^{2}-16 a d b c<0 \\
& \Leftrightarrow a^{2} d^{2}<a d b c \\
& \Leftrightarrow a d(a d-b c)<0 \\
& \Leftrightarrow a d<0 .
\end{aligned}
$$

Hence, with $(a, b, c, d)=(-10-\lambda,-9,10,6-\lambda)$ we find $F$ and it satisfies the condition on $a, b, c$, and $d$. 


\section{Appendix B. Results Relative to the Affine Kernel}

In this section, it is gathered the extension to the affine kernel (19) of the propositions proposed in Sections 3 and 4 when considering the exponential kernel (8).

\section{B.1. Boundary Control Synthesis}

Theorem B.1. If there exist $\nu>0, \mu$ in $(-1,1)$, a matrix $U$ in $\mathbb{R}^{q \times n}$, and symmetric positive definite matrices $S^{-}$in $\mathbb{R}^{m \times m}, S^{+}$in $\mathbb{R}^{(n-m) \times(n-m)}$ such that, with $S(x)=\operatorname{diag}\left[(1+\mu x)^{-1} S^{-},(1-\mu x) S^{+}\right]$, the following conditions hold, for all $x \in[0,1]$,

$$
\begin{aligned}
& S(x) \Lambda(x)=\Lambda(x) S(x), \\
& -2 \mu S(0)+S(x) F^{\top}(x)|\Lambda(x)|^{-1}+|\Lambda(x)|^{-1} F(x) S(x) \leq-2 \nu S(x)|\Lambda(x)|^{-1}, \\
& {\left[\begin{array}{cc}
\operatorname{diag}\left[S^{-},(1-\mu) S^{+}\right] & (T S(0)+L U)^{\top} \\
T S(0)+L U & \operatorname{diag}\left[(1+\mu)^{-1} S^{-}, S^{+}\right]
\end{array}\right] \geq 0,}
\end{aligned}
$$

then the boundary control given by (25) with

$$
K_{B}=U S^{-1}(0)
$$

is such that system (1)-(3) is GES and a Lyapunov function of the form (7) exists with $\mathcal{Q}(x)=S^{-1}(x)$.

Proof. The proof is similar to the one of Theorem 3.1. Replacing $U$ by $K_{B} S(0)$ and applying the Schur complement formula in (B.3) one gets

$$
\operatorname{diag}\left[S^{-},(1-\mu) S^{+}\right]-S(0)\left(T+L K_{B}\right)^{\top} \operatorname{diag}\left[(1+\mu)^{-1} S^{-}, S^{+}\right]^{-1}\left(T+L K_{B}\right) S(0) \geq 0 .
$$

Reassembling the term in one matrix and multiplying from the left and right by $S^{-1}(0)$ we get the matrix

$$
\tilde{M}=\left[\begin{array}{ll}
\tilde{M}_{--} & \tilde{M}_{-+} \\
\tilde{M}_{+-} & \tilde{M}_{++}
\end{array}\right] \geq 0
$$

with

$$
\begin{aligned}
\tilde{M}_{--}= & \left(S^{-}\right)^{-1}-(1+\mu)\left(T+L K_{B}\right)_{--}^{\top}\left(S^{-}\right)^{-1}\left(T+L K_{B}\right)_{--}-\left(T+L K_{B}\right)_{+-}^{\top}\left(S^{+}\right)^{-1}\left(T+L K_{B}\right)_{+-}, \\
\tilde{M}_{-+}= & -(1+\mu)\left(T+L K_{B}\right)_{--}^{\top}\left(S^{-}\right)^{-1}\left(T+L K_{B}\right)_{-+}-\left(T+L K_{B}\right)_{+-}^{\top}\left(S^{+}\right)^{-1}\left(T+L K_{B}\right)_{++}, \\
\tilde{M}_{+-}= & \tilde{M}_{-+}^{\top}, \\
\tilde{M}_{++}= & (1-\mu)\left(S^{+}\right)^{-1}-(1+\mu)\left(T+L K_{B}\right)_{-+}^{\top}\left(S^{-}\right)^{-1}\left(T+L K_{B}\right)_{-+} \\
& -\left(T+L K_{B}\right)_{++}^{\top}\left(S^{+}\right)^{-1}\left(T+L K_{B}\right)_{++} .
\end{aligned}
$$

Letting $Q^{-}=\left(S^{-}\right)^{-1}, Q^{+}=\left(S^{+}\right)^{-1}$ we get

- condition (20) by multiplying left and right (B.1) by $\mathcal{Q}(x)$,

- condition (21) by multiplying left and right (B.2) by $\mathcal{Q}(x)$,

- condition (22) from the matrix $\tilde{M}$ in (B.6).

It concludes the proof of Theorem B.1. 


\section{B.2. Distributed Control Synthesis}

Henceforth, for a given $\mu$ in $(-1,1)$ let us denote $I_{n, m}^{a}(x)$ the matrix

$$
I_{n, m}^{a}(x)=\operatorname{diag}\left[(1+\mu x) I_{m},(1-\mu x) I_{n-m}\right]
$$

Theorem B.2. Let an integer $\ell>0$ be given. If there exist $\nu>0, \mu$ in $(-1,1)$, matrices $U_{i}$ in $\mathbb{R}^{p \times n}$, $i=1, \ldots, \ell$, and symmetric positive definite matrices $S^{-}$in $\mathbb{R}^{m \times m}, S^{+}$in $\mathbb{R}^{(n-m) \times(n-m)}$ such that, with $S(x)=\operatorname{diag}\left[(1+\mu x)^{-1} S^{-},(1-\mu x)^{-1} S^{+}\right]$, the following conditions hold, for all $x \in[0,1]$,

$$
\begin{aligned}
& S(x) \Lambda(x)=\Lambda(x) S(x) \\
& \left(|\Lambda(x)|^{-1} H(x)-\mu I_{n}\right) S(x)+S(x)\left(|\Lambda(x)|^{-1} H(x)-\mu I_{n}\right)^{\top} \\
& +\sum_{i=1}^{\ell} \alpha_{i}(x)\left(I_{n, m}^{a}(x)\right)^{-1} U_{i}^{\top} B^{\top}(x)|\Lambda(x)|^{-1}+\sum_{i=1}^{\ell} \alpha_{i}(x)|\Lambda(x)|^{-1} B(x) U_{i}\left(I_{n, m}^{a}(x)\right)^{-1} \\
& \leq-2 \nu S(x)|\Lambda(x)|^{-1}, \\
& {\left[\begin{array}{c}
\operatorname{diag}\left[S^{-},(1-\mu) S^{+}\right] \\
G S(0)
\end{array} \quad \operatorname{diag}\left[(1+\mu)^{-1} S^{-}, S^{+}\right]\right] \geq 0,}
\end{aligned}
$$

with $I_{n, m}^{a}(x)$ given in (B.7), then the distributed control given by (32) and (33) with

$$
K_{i}=U_{i} S^{-1}(0), \quad i=1, \ldots, \ell
$$

is such that system (1)-(3) is GES and a Lyapunov function of the form (7) exists with $\mathcal{Q}(x)=S^{-1}(x)$.

Proof. The proof of Theorem B.2 is immediate with the proof of Theorems B.1 and 3.4.

\section{B.3. Overapproximation: $\Lambda$ and $F$ constants}

Conditions of Proposition 2.5 can be easily verified. For fixed $\mu$ in $(-1,1), Q^{-}$in $\mathbb{R}^{m \times m}, Q^{+}$in $\mathbb{R}^{(n-m) \times(n-m)}$, we write

$$
\begin{aligned}
& Q_{0}=\operatorname{diag}\left[Q^{-}, Q^{+}\right] \\
& Q_{1}=\operatorname{diag}\left[(1+\mu) Q^{-},(1-\mu) Q^{+}\right]
\end{aligned}
$$

and we have the following proposition.

Proposition B.3. If there exist $\nu \in \mathbb{R}^{+}, \mu \in(-1,1)$, and symmetric positive definite matrices $Q^{-}$in $\mathbb{R}^{m \times m}$ and $Q^{+}$in $\mathbb{R}^{(n-m) \times(n-m)}$ such that

$$
\begin{aligned}
& Q_{0} \Lambda=\Lambda Q_{0} \\
& Q_{1} \Lambda=\Lambda Q_{1} \\
& -\mu Q_{0}+F^{\top}|\Lambda|^{-1} Q_{0}+Q_{0}|\Lambda|^{-1} F \leq-2 \nu|\Lambda|^{-1} Q_{0}, \\
& -\mu Q_{0}+F^{\top}|\Lambda|^{-1} Q_{1}+Q_{1}|\Lambda|^{-1} F \leq-2 \nu|\Lambda|^{-1} Q_{1},
\end{aligned}
$$

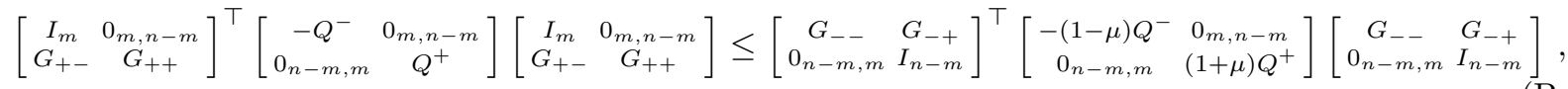


then conditions (20), (21), and (22) are satisfied for all $x \in[0,1]$.

Proof. Note that $\mathcal{Q}(x)$ satisfies

$$
\begin{aligned}
\mathcal{Q}(x) & =\operatorname{diag}\left[(1+\mu x) Q^{-},(1-\mu x) Q^{+}\right] \\
& =(1-x) Q_{0}+x Q_{1} .
\end{aligned}
$$

Since $1-x($ resp. $x)$ is positive and $Q_{0}\left(\right.$ resp. $\left.Q_{1}\right)$ verifies (B.16) (resp. (B.17)) then $(1-x) Q_{0}$ (resp. $\left.x Q_{1}\right)$ verifies also (B.16) (resp. (B.17)). Hence, condition (21) holds. In the same manner condition (20) holds. The verification of (22) is immediate since (B.18) is equivalent. It concludes the proof of Proposition B.3.

Similarly to what have been done with the exponential kernel (8), we can find some tractable conditions for the checking of conditions of Theorems B.1 and B.2. For fixed $\mu$ in $(-1,1), S^{-}$in $\mathbb{R}^{n \times n}, S^{+}$in $\mathbb{R}^{(n-m) \times(n-m)}$, we write

and for $i, j=0,1$,

$$
\mathcal{S}(x)=\operatorname{diag}\left[\left[(1+\mu x)^{-1} S^{-},(1-\mu x)^{-1} S^{+}\right], \quad x \in[0,1],\right.
$$

$$
\begin{aligned}
\tilde{S}_{i j} & =\operatorname{diag}\left[(1+\mu i)^{-1} S^{-},(1-\mu j)^{-1} S^{+}\right], \\
\hat{S}_{i j} & =\operatorname{diag}\left[(1+\mu i)^{-1} S^{-},(1-\mu j) S^{+}\right], \\
\hat{I}_{n, m}^{i j} & =\operatorname{diag}\left[(1+\mu i) I_{m},(1-\mu j) I_{n-m}\right] .
\end{aligned}
$$

Since (B.19) is no more affine in the $\left(S^{-}, S^{+}\right)$-plane we need the following lemma.

Lemma B.4. For all $x \in[0,1], \mathcal{S}(x)$ lies in the convex hull of $\left\{\tilde{S}_{00}, \tilde{S}_{10}, \tilde{S}_{11}\right\}$ if $\mu>0$ and of $\left\{\tilde{S}_{00}, \tilde{S}_{01}, \tilde{S}_{11}\right\}$ if $\mu<0$.

Proof. We assume that $\mu>0$, the case $\mu<0$ is analogous. The map $\mathcal{S}(x)$ is a parametrized curve in the $\left(S^{-}, S^{+}\right)$-plane. We can express it as an explicit curve. We have $(1+\mu x)^{-1}(1-\mu x)^{-1}=\left(1-\mu^{2} x^{2}\right)^{-1}$, thus the expression of the explicit curve is given by

$$
\tilde{h}(X)=\frac{X}{2 X-1}, \quad X \in \tilde{\rho}=\left[(1+\mu)^{-1}, 1\right] .
$$

This curve is convex on this interval. Then,

$$
\tilde{h}\left(\alpha(1+\mu)^{-1}+(1-\alpha)\right) \leq \alpha \tilde{g}\left((1+\mu)^{-1}\right)+(1-\alpha) \tilde{g}(1), \quad \alpha \in(0,1),
$$

where $\tilde{g}(X)=\left(\frac{(1+\mu)^{-1}-1}{(1-\mu)^{-1}-1}\right) X+(1+\mu)^{-1}+1$. When $X$ lies in $\tilde{\rho}, \tilde{g}(X)$ describes the straight line between $\tilde{S}_{00}$ and $\tilde{S}_{11}$. Hence, for $X \in \tilde{\rho}$ one has $\tilde{h}(X) \leq \tilde{g}(X)$. Thus, $\mathcal{S}$ lies in the convex hull of $\left\{\tilde{S}_{00}, \tilde{S}_{10}, \tilde{S}_{11}\right\}$.

Proposition B.5. If there exist $\nu>0, \mu$ in $(-1,0) \cup(0,1)$, a matrix $U$ in $\mathbb{R}^{q \times n}$, and symmetric matrices $S^{-}$ in $\mathbb{R}^{m \times m}, S^{+}$in $\mathbb{R}^{(n-m) \times(n-m)}$ such that the following conditions hold,

$$
\begin{aligned}
& \tilde{S}_{i j} \Lambda=\Lambda \tilde{S}_{i j}, \\
& -2 \mu \tilde{S}_{i j}+\tilde{S}_{i j} F^{\top}|\Lambda|^{-1}+|\Lambda|^{-1} F \tilde{S}_{i j} \leq-2 \nu \tilde{S}_{i j}|\Lambda|^{-1}, \\
& {\left[\begin{array}{cc}
\hat{S}_{01} & \left(T \hat{S}_{00}+L U\right)^{\top} \\
T \hat{S}_{00}+L U & \hat{S}_{10}
\end{array}\right] \geq 0,}
\end{aligned}
$$


for $(i, j) \in\{(0,0),(1,0),(1,1)\}$ if $\mu>0$ and for $(i, j) \in\{(0,0),(0,1),(1,1)\}$ if $\mu<0$, then conditions (B.1), (B.3), and (B.2) are satisfied for all $x \in[0,1]$.

Proof. We have that

- the inequality (B.25) is equivalent to (B.3),

- by Lemma B.4 the constraint of equality (B.1) and LMI (B.3) are embedded in the polytope built by the points $\tilde{S}_{00}, \tilde{S}_{10}$, and $\tilde{S}_{11}$ if $\mu>0$ or by the points $\tilde{S}_{00}, \tilde{S}_{01}$, and $\tilde{S}_{11}$ if $\mu<0$.

Thus, conditions (B.1), (B.2), and (B.3) are satisfied for all $x \in[0,1]$. The proof of Proposition B.5 is complete.

Proposition B.6. Let an integer $\ell>0$ be given. If there exist $\nu>0, \mu$ in $(-1,0) \cup(0,1)$, matrices $U_{i}$ in $\mathbb{R}^{p \times n}, i=1, \ldots, \ell$, and symmetric positive definite matrices $S^{-}$in $\mathbb{R}^{m \times m}, S^{+}$in $\mathbb{R}^{(n-m) \times(n-m)}$ such that the following conditions hold,

$$
\begin{aligned}
& \tilde{S}_{j k} \Lambda=\Lambda \tilde{S}_{j k}, \\
& \left(|\Lambda|^{-1} H-\mu I_{n}\right) \tilde{S}_{j k}+\tilde{S}_{j k}\left(|\Lambda|^{-1} H-\mu I_{n}\right)^{\top} \\
& +\sum_{i=1}^{\ell} \min _{x \in[0,1]} \alpha_{i}(x)\left(\hat{I}_{n, m}^{j k}\right)^{-1} U_{i}^{\top} B^{\top}|\Lambda|^{-1}+\sum_{i=1}^{\ell} \min _{x \in[0,1]} \alpha_{i}(x)|\Lambda|^{-1} B U_{i}\left(\hat{I}_{n, m}^{j k}\right)^{-1} \leq-2 \nu \tilde{S}_{j k}|\Lambda|^{-1}, \\
& \left(|\Lambda|^{-1} H-\mu I_{n}\right) \tilde{S}_{j k}+\tilde{S}_{j k}\left(|\Lambda|^{-1} H(x)-\mu I_{n}\right)^{\top} \\
& +\sum_{i=1}^{\ell} \max _{x \in[0,1]} \alpha_{i}(x)\left(\hat{I}_{n, m}^{j k}\right)^{-1} U_{i}^{\top} B^{\top}|\Lambda|^{-1}+\sum_{i=1}^{\ell} \max _{x \in[0,1]} \alpha_{i}(x)|\Lambda|^{-1} B U_{i}\left(\hat{I}_{n, m}^{j k}\right)^{-1} \leq-2 \nu \tilde{S}_{j k}|\Lambda|^{-1}, \\
& {\left[\begin{array}{c}
\hat{S}_{01}\left(G \hat{S}_{00}\right)^{\top} \\
G \hat{S}_{00} \\
\hat{S}_{10}
\end{array}\right] \geq 0,}
\end{aligned}
$$

for $(j, k) \in\{(0,0),(1,0),(1,1)\}$ if $\mu>0$ and for $(j, k) \in\{(0,0),(0,1),(1,1)\}$ if $\mu<0$, then conditions (B.8), (B.9), and (B.10) are satisfied for all $x \in[0,1]$.

Proof. First of all the maximum and the minimum of $\alpha_{i}, i=1, \ldots, \ell$, are well-defined since $\alpha_{i}$ are continuous functions defined in the compact $[0,1]$. We have that

- the inequality (B.29) corresponds to (B.10),

- by Lemma B.4 the constraint of equality (B.8) and LMI (B.9) are embedded in the polytope built by the points $\tilde{S}_{00}, \tilde{S}_{10}$, and $\tilde{S}_{11}$ if $\mu>0$ or by the points $\tilde{S}_{00}, \tilde{S}_{01}$, and $\tilde{S}_{11}$ if $\mu<0$.

Thus, conditions (B.8), (B.9), and (B.10) are satisfied for all $x \in[0,1]$. The proof of Proposition B.6 is complete. 


\section{B.4. Overapproximation: $\Lambda$ and $F$ spatially-varying}

Similar results may be stated for the stability conditions and controller design conditions whenever $\Lambda$ and $F$ are spatially varying. We are considering the sets $\mathcal{W}, \mathcal{R}$, and $\mathcal{Z}$ defined in (55), (65), and (66) respectively. Let us remind that, for all $i=1, \ldots, N, W_{i}$ are in $\mathcal{W}$, for all $j=1, \ldots, M_{1}, R_{j}$ are in $\mathcal{R}$, and for all $k=1, \ldots, M_{2}$, $Z_{k}$ are in $\mathcal{Z}$.

Proposition B.7. If there exist $\nu>0, \mu$ in $(-1,1)$, and diagonal positive definite matrices $Q^{-}$in $\mathbb{R}^{m \times m}$ and $Q^{+}$in $\mathbb{R}^{(n-m) \times(n-m)}$ such that

$$
\begin{gathered}
-\mu Q_{0}+W_{i}^{\top} Q_{0}+Q_{0} W_{i} \leq-2 \nu|\bar{\Lambda}|^{-1} Q_{0} \\
-\mu Q_{0}+W_{i}^{\top} Q_{1}+Q_{1} W_{i} \leq-2 \nu|\bar{\Lambda}|^{-1} Q_{1}, \\
{\left[\begin{array}{cc}
I_{m} & 0_{m, n-m} \\
G_{+-} & G_{++}
\end{array}\right]^{\top}\left[\begin{array}{cc}
-Q^{-} & 0_{m, n-m} \\
0_{n-m, m} & Q^{+}
\end{array}\right]\left[\begin{array}{cc}
I_{m} & 0_{m, n-m} \\
G_{+-} & G_{++}
\end{array}\right] \leq\left[\begin{array}{cc}
G_{--} & G_{-+} \\
0_{n-m, m} & I_{n-m}
\end{array}\right]^{\top}\left[\begin{array}{cc}
-(1-\mu) Q^{-} & 0_{m, n-m} \\
0_{n-m, m} & (1+\mu) Q^{+}
\end{array}\right]} \\
\times\left[\begin{array}{ccc}
G-- & G_{-+} \\
0_{n-m, m} & I_{n-m}
\end{array}\right],
\end{gathered}
$$

for all $i=1, \ldots, N$, where $|\bar{\Lambda}|$ is defined in (58) and $Q_{0}, Q_{1}$ are respectely defined in (B.12), (B.13), then conditions (20), (21), and (22) are satisfied for all $x \in[0,1]$.

Proof. The proof is similar to the proof of Proposition 4.7. Multiplying (B.30) and (B.31) by $\alpha_{i}$ and making the sum for $i=1, \ldots, N$, we get

$$
\begin{aligned}
& -\mu Q_{0}+W(x)^{\top} Q_{0}+Q_{0} W(x) \leq-2 \nu|\bar{\Lambda}|^{-1} Q_{0} \\
& -\mu Q_{0}+W(x)^{\top} Q_{1}+Q_{1} W(x) \leq-2 \nu|\bar{\Lambda}|^{-1} Q_{1}
\end{aligned}
$$

for all $x \in[0,1]$.

- Using the fact that

$$
\mathcal{Q}(x)=(1-x) Q_{0}+x Q_{1}
$$

and the definition of $\bar{\Lambda}$ in (58), one gets $(21)$.

- Condition (20) is automatically satisfied because of the diagonal form of $Q^{-}$and $Q^{+}$.

The proof of Proposition B.7 is complete.

Proposition B.8. If there exist $\nu>0, \mu$ in $(-1,0) \cup(0,1)$, a matrix $U$ in $\mathbb{R}^{q \times n}$, and diagonal positive definite matrices $S^{-}$in $\mathbb{R}^{m \times m}, S^{+}$in $\mathbb{R}^{(n-m) \times(n-m)}$ such that, the following conditions hold,

$$
\begin{aligned}
& -2 \mu \tilde{S}_{j k}+\tilde{S}_{j k} W_{i}^{\top}+W_{i} \tilde{S}_{j k} \leq-2 \nu \tilde{S}_{j k}|\bar{\Lambda}|^{-1} \\
& {\left[\begin{array}{c}
\hat{S}_{01} \\
T \hat{S}_{00}+L U
\end{array} \quad \begin{array}{c}
\left(T \hat{S}_{00}+L U\right)^{\top} \\
\hat{S}_{10}
\end{array}\right] \geq 0,}
\end{aligned}
$$

for all $i=1, \ldots, N$, and $(j, k) \in\{(0,0),(1,0),(1,1)\}$ if $\mu>0,(j, k) \in\{(0,0),(0,1),(1,1)\}$ if $\mu<0$, then conditions (B.1), (B.2), and (B.3) are satisfied for all $x \in[0,1]$.

Proof. As in the proofs of Proposition 4.7 and 4.9 we multiply (B.35) by $\alpha_{i}$, and make the sum for $i=1, \ldots, N$, getting

$$
-2 \mu \tilde{S}_{j k}+\tilde{S}_{j k} W(x)^{\top}+W(x) \tilde{S}_{j k} \leq-2 \nu \tilde{S}_{j k}|\bar{\Lambda}|^{-1}
$$

for all $x \in[0,1],(j, k) \in\{(0,0),(1,0),(1,1)\}$ if $\mu>0,(j, k) \in\{(0,0),(0,1),(1,1)\}$ if $\mu<0$.

- Using Lemma B.4 and the definition of $\bar{\Lambda}$ in (58), one gets (B.2).

- Condition (B.1) is automatically satisfied because of the diagonal form of $S^{-}$and $S^{+}$. 
- Condition (B.3) is equivalent to condition (B.36).

The proof of Proposition B.8 is complete.

Proposition B.9. Let an integer $\ell>0$ be given. If there exist $\nu>0, \mu$ in $(-1,0) \cup(0,1)$, matrices $U_{i}$ in $\mathbb{R}^{p \times n}$, $i=1, \ldots, \ell$, and diagonal positive definite matrices $S^{-}$in $\mathbb{R}^{m \times m}, S^{+}$in $\mathbb{R}^{(n-m) \times(n-m)}$ such that the following conditions hold

$$
\begin{aligned}
& \left(R_{\iota_{1}}-\mu I_{n}\right) S_{j k}+S_{j k}\left(R_{\iota_{1}}-\mu I_{n}\right)^{\top} \\
& +\sum_{i=1}^{\ell} \min _{x \in[0,1]} \alpha_{i}(x)\left(\hat{I}_{n, m}^{j k}\right)^{-1} U_{i}^{\top} Z_{\iota_{2}}^{\top}+\sum_{i=1}^{\ell} \min _{x \in[0,1]} \alpha_{i}(x) Z_{\iota_{2}} U_{i}\left(\hat{I}_{n, m}^{j k}\right)^{-1} \leq-2 \nu S_{j k}|\bar{\Lambda}|^{-1}, \\
& \left(R_{\iota_{1}}-\mu I_{n}\right) S_{j k}+S_{j k}\left(R_{\iota_{1}}-\mu I_{n}\right)^{\top} \\
& +\sum_{i=1}^{\ell} \max _{x \in[0,1]} \alpha_{i}(x)\left(\hat{I}_{n, m}^{j k}\right)^{-1} U_{i}^{\top} Z_{\iota_{2}}^{\top}+\sum_{i=1}^{\ell} \max _{x \in[0,1]} \alpha_{i}(x) Z_{\iota_{2}} U_{i}\left(\hat{I}_{n, m}^{j k}\right)^{-1} \leq-2 \nu S_{j k}|\bar{\Lambda}|^{-1}, \\
& {\left[\begin{array}{c}
\hat{S}_{01}\left(G \hat{S}_{00}\right)^{\top} \\
G \hat{S}_{00} \\
\hat{S}_{10}
\end{array}\right] \geq 0}
\end{aligned}
$$

\begin{tabular}{|c|c|c|c|}
\hline \multirow{2}{*}{\multicolumn{2}{|c|}{ Stability Analysis }} & Non Spatially Varying & Proposition B.3 \\
\hline & & Spatially Varying & Proposition B.7 \\
\hline \multirow{4}{*}{ Controller Synthesis } & \multirow{2}{*}{ Boundary Control } & Non Spatially Varying & Proposition B.5 \\
\hline & & Spatially Varying & Proposition B.8 \\
\hline & \multirow{2}{*}{ Distributed Control } & Non Spatially Varying & Proposition B.6 \\
\hline & & Spatially Varying & Proposition B.9 \\
\hline
\end{tabular}

for all $\iota_{1}=1, \ldots, M_{1}, \iota_{2}=1, \ldots, M_{2}$, and $(j, k) \in\{(0,0),(1,0),(1,1)\}$ if $\mu>0,(j, k) \in\{(0,0),(0,1),(1,1)\}$ if $\mu<0$, then conditions (B.8), (B.9), and (B.10) are satisfied for all $x \in[0,1]$.

Proof. Using Lemma B.4 and the proof of Proposition 4.10 the result follows.

As for Section 4 we propose a summary table for the results of Appendix B.

TABLE 2. Summary table of the results of Appendix B relative to the affine kernel (19). 